hep-ph/9605398

May 1996

\title{
Search for supersymmetry with a light gravitino at the Fermilab Tevatron and CERN LEP colliders
}

\author{
S. Ambrosanio*円, G. L. Kanef, Graham D. Kribs], Stephen P. Martinf, \\ Randall Physics Laboratory, University of Michigan, \\ Ann Arbor, MI 48109-1120 \\ S. Mrennap \\ High Energy Physics Division, Argonne National Laboratory, \\ Argonne, IL 60439
}

\begin{abstract}
We analyze the prospects for discovering supersymmetry at the Fermilab Tevatron and CERN LEP colliders in the scenario that the lightest supersymmetric particle is a gravitino of mass $\lesssim 1 \mathrm{keV}$. We consider in particular the case that the lightest neutralino has a nearly $100 \%$ branching fraction into gravitino + photon within the detector. This implies that supersymmetric events should contain both missing (transverse) energy and two energetic photons. Therefore one can search for supersymmetry simply through inclusive production of superpartners. We consider the exclusion and reach capabilities of the Tevatron in exploring the supersymmetric parameter space, and study the efficiencies which can be achieved in this search. We also consider the discovery reach and backgrounds at LEP with $\sqrt{s}=160,175$, and $190 \mathrm{GeV}$.
\end{abstract}

*Supported mainly by an INFN postdoctoral fellowship, Italy.

\footnotetext{
${ }^{1}$ ambros@umich.edu

2 gkane@umich.edu

${ }^{3}$ kribs@umich.edu

${ }^{4}$ spmartin@umich.edu

${ }^{5}$ mrenna@hep.anl.gov
} 


\section{Introduction}

One of the intriguing theoretical aspects of supersymmetry is that if it is realized as a local symmetry, it necessarily and automatically incorporates gravity. This connection is of no consequence in most studies of supersymmetric phenomenology at colliders, because of the familiar negligibility of gravitational interactions. However, this need not be so if the gravitino (the spin $3 / 2$ partner of the graviton) is very light. The gravitino $(\tilde{G})$ obtains its mass by absorbing the spin $1 / 2$ would-be goldstino associated with the spontaneous breaking of supersymmetry. In the high-energy limit, the interactions of the $\pm 1 / 2$ helicity components of the gravitino are the same as those of the goldstino it has absorbed. As emphasized originally by Fayet [1], these interactions are proportional to $1 / m_{\tilde{G}}$ in the $m_{\tilde{G}} \rightarrow 0$ limit and are therefore potentially important even for processes at ordinary energies.

However, the strength of gravitino interactions (or equivalently $1 / m_{\tilde{G}}$ ) certainly cannot be arbitrarily large. The gravitino mass is related to the scale of spontaneous supersymmetry breaking by

$$
m_{\tilde{G}}=\frac{\Lambda_{\mathrm{SUSY}}^{2}}{\sqrt{3} M}=5.9 \times 10^{-5}\left(\frac{\Lambda_{\mathrm{SUSY}}}{500 \mathrm{GeV}}\right)^{2} \mathrm{eV} .
$$

Here $M=\left(8 \pi G_{\text {Newton }}\right)^{-1 / 2}=2.4 \times 10^{18} \mathrm{GeV}$, and $\Lambda_{\mathrm{SUSY}}^{-2}$ is the coupling of the would-be goldstino to the divergence of the supercurrent. Now, the scale $\Lambda_{\mathrm{SUSY}}$ should at least exceed the mass of the heaviest of the superpartners of the Standard Model (SM) particles, typically a gluino $(\tilde{g})$ or squark $(\tilde{q})$, and probably greatly so. [One might expect a significant hierarchy between $\Lambda_{\text {SUSY }}$ and the electroweak scale, in order that negative radiative corrections to the Higgs scalar (mass) ${ }^{2}$ can be effective in driving electroweak symmetry breaking.] Thus if one takes e.g. a bound $\Lambda_{\mathrm{SUSY}}>500 \mathrm{GeV}$, equation (11) becomes a lower bound on the gravitino mass of roughly $6 \times 10^{-5} \mathrm{eV}$. In any case a given mass spectrum for the sparticles always implies a lower bound on $m_{\tilde{G}}$. This is equivalent to a bound on the strength of the gravitino's interactions with the SM particles and their superpartners. This type of bound is quite conservative, and is certainly not expected to be saturated in particular models [2, 3] of supersymmetry-breaking at low energies. For example, recently proposed models [3] of dynamical supersymmetry breaking communicated to the visible sector by gauge interactions evidently favor $m_{\tilde{G}} \gtrsim 1 \mathrm{eV}$ which automatically avoids a dangerous R-axion [4], although other ways of doing this might be possible.

One can also attempt to obtain a lower bound on the gravitino mass by examining the requirements of partial-wave unitarity for, e.g., the scattering of two gluons into two gravitinos [5]. That process has contributions at tree-level from t- and u-channel exchanges of the gluino. 
As shown in ref. [5], tree-level partial wave unitarity is violated in this process when $\sqrt{s}$ exceeds

$$
E_{\text {crit }}=\sqrt{288 \pi} M m_{\tilde{G}} / m_{\tilde{g}}
$$

Now, one way to interpret this result is that the gravitino interactions, being gravitational, should not become strong below, say, the scale $M$; then (2) would become a lower bound on the gravitino mass of $m_{\tilde{G}} \gtrsim m_{\tilde{g}} / 30$. If this were true, the gravitino would always interact far too weakly to play any role in collider experiments. However, it seems preferable to interpret the critical energy indicated by (2) as the maximum value of a scale $\Lambda_{\tilde{g}}$ of unknown new physics at which $m_{\tilde{g}}$ becomes effective. In that case, one finds only that $m_{\tilde{G}} \gtrsim \Lambda_{\tilde{g}} m_{\tilde{g}} / 30 M$. If $\Lambda_{\tilde{g}}$ is smaller than the ultimate scale of supersymmetry breaking $\Lambda_{\mathrm{SUSY}}$, this constraint is vacuous when compared with (11).

On the other hand, cosmological constraints [6] seem to place an upper bound on $m_{\tilde{G}}$ of about $10^{4} \mathrm{eV}$, at least in the absence of late inflation. There is then still a window of perhaps 9 orders of magnitude for the mass of a light gravitino. In particular classes of models, this window can be much smaller. Throughout this window, $m_{\tilde{G}}$ is clearly insignificant for collider kinematics, and so can be taken to simply parameterize the strength of the gravitino's interactions.

Most collider phenomenology studies performed up to now assume that the lightest supersymmetric particle (LSP) is a neutralino (mixture of neutral higgsinos and $S U(2)_{L} \times U(1)_{Y}$ gauginos). If, as is most often assumed, $R$-parity is exactly conserved, then supersymmetric particles will always be produced in pairs, and the LSP is absolutely stable. In this "neutralino LSP scenario", every supersymmetric event will feature two LSPs leaving the detector. Therefore the signals for supersymmetry always involve missing energy, often together with lepton and/or multi-jet signatures corresponding to particular decay chains of the superpartners produced [7]. In the "gravitino LSP scenario", however, the signatures should be quite different if the decays of superpartners into gravitinos occur within the detector a significant fraction of the time.

For example, in the most obvious case that the next-to-lightest supersymmetric particle (NLSP) is a neutralino $\left(\tilde{N}_{1}\right)$ with a non-zero photino component, one has the interesting decay [1, 8

$$
\tilde{N}_{1} \rightarrow \gamma \tilde{G}
$$

In the rest frame of the decaying $\tilde{N}_{1}$, the photon takes an energy equal to $m_{\tilde{N}_{1}} / 2$ and is produced isotropically. The photons produced in supersymmetric events should therefore often be energetic enough to pass cuts designed to reduce SM backgrounds. The gravitino still carries away a significant amount of missing energy. Thus, supersymmetric signals in the gravitino LSP 
scenario should be similar to those in the neutralino LSP scenario but with two (one) energetic, often isolated, photons if both (one) of the $\tilde{N}_{1}$ decays occur within the detector. Several recent papers have presented interesting studies of this type of signal at the Next Linear Collider [9], er colliders [10], and the Tevatron [11].

Since the presence of additional energetic photons in supersymmetric events would provide a welcome and powerful discriminant against SM backgrounds, it is possible to consider supersymmetry discovery signals based on inclusive production of all superpartners. The signatures in the gravitino LSP case are $\gamma \gamma \mathbb{E}_{T}+X$ (or possibly $\gamma \mathbb{E}_{T}+X$ ), where $X$ is an arbitrary collection of leptons + jets (including $X=$ nothing which can occur e.g. in the cases of $\tilde{N}_{1} \tilde{N}_{1}$ or $\tilde{\nu} \tilde{\nu}$ production). The purpose of the present paper is to study this strategy at the Fermilab Tevatron and CERN LEP colliders in the Minimal Supersymmetric Standard Model (MSSM) with a light gravitino. We define the MSSM to be the minimal supersymmetric extension of the SM with $R$-parity conserved. Squarks (other than the top squarks) are assumed to be very nearly degenerate in mass, as suggested both by theory and the absence of flavor-changing neutral currents in experiment. Sleptons with the same electroweak quantum numbers are also assumed to be degenerate in mass. We will also often, but not always, make use of the "gaugino mass unification" assumption for running gaugino mass parameters:

$$
M_{2}=\frac{3}{5 \tan ^{2} \theta_{W}} M_{1}=\frac{\alpha_{2}}{\alpha_{3}} M_{3}
$$

which arises both in gauge-mediated and gravity-mediated supersymmetry breaking.?

This study is motivated in part by the observation at CDF of a single ee $\gamma \gamma \mathbb{E}_{T}$ event [12] that does not seem to have a SM interpretation. It has already been suggested that this event can be explained by supersymmetry, either in the gravitino LSP scenario [13, 14] considered here or in a scenario with a higgsino-like neutralino LSP [14]. In [14], we found that if this event is due to selectron pair production followed by the decays $\tilde{e} \rightarrow e \tilde{N}_{1}$ and $\tilde{N}_{1} \rightarrow \gamma \tilde{G}$, then the kinematic requirements of the event place rough bounds of $80 \mathrm{GeV}<m_{\tilde{e}}$ and $38 \mathrm{GeV}<m_{\tilde{N}_{1}}<$ $100 \mathrm{GeV}$. However, this event can also be ascribed to pair production of charginos, as we shall remark below. In any case, we will maintain a more general point of view in most of the present work, rather than restrict our attention to the parameter space suggested by that one event.

This paper is organized as follows. In section 2, we will consider the decays of supersymmetric particles into 2- and 3-body final states which include a gravitino. The 2-body decays $\tilde{N}_{1} \rightarrow Z \tilde{G}$ and $\tilde{N}_{1} \rightarrow h^{0} \tilde{G}$ (which might compete with $\tilde{N}_{1} \rightarrow \gamma \tilde{G}$ if the photino component of $\tilde{N}_{1}$ is very small) turn out to be subject to a very strong kinematic suppression. We also discuss expectations for the decay lengths of $\tilde{N}_{1}$, and note the existence of regions of parameter

\footnotetext{
${ }^{1}$ In particular, this "unification" relation can be well-motivated even in the absence of gauge-coupling unification at a very high energy scale; see e.g. [3].
} 
space where the decay $\tilde{N}_{1} \rightarrow \gamma \tilde{G}$ is unduly suppressed. In section 3 , we consider the limits on the reach of the Tevatron by studying the cross-sections for inclusive sparticle production. These rates are more interesting than in the case of the neutralino LSP scenario because of the relative ease with which arbitrary types of sparticle production can be detected using energetic photons. We propose a set of cuts designed to maximize the efficiency for detection of supersymmetry at the Tevatron via the signature $\gamma \gamma \mathbb{E}_{T}+X$, and study the efficiencies and lepton and jet multiplicities obtained using several sets of model parameters as test cases. In section 4 we discuss the possibility of discovering supersymmetry at LEP with $\sqrt{s}=160,175$, and 190 $\mathrm{GeV}$, including an analysis of the relevant backgrounds. Section 5 contains some concluding remarks.

\section{Decays into gravitinos}

The decay $\tilde{N}_{1} \rightarrow \gamma \tilde{G}$ will play the central role in the phenomenological discussions to follow. In sections 3 and 4 we will simply assume that this decay is the only important one involving the gravitino, and that it always occurs within the detector. However, it is interesting to consider other possible decays which might have an impact on collider phenomenology as well. If $\tilde{N}_{1}$ is the NLSP and is fairly heavy, (but especially if it is beyond the reach of the Tevatron with the present integrated luminosity), the decays $\tilde{N}_{1} \rightarrow h^{0} \tilde{G}$ and $\tilde{N}_{1} \rightarrow Z \tilde{G}$ can at least naively be important. Also it is interesting to consider the possibility that a sparticle other than $\tilde{N}_{1}$ is the NLSP. Finally, if $m_{\tilde{G}}$ is very small $(\ll 1 \mathrm{eV})$ one can even entertain the possibility that superpartners other than the NLSP can decay directly into final states containing a gravitino. In this section we present general formulas for the decay widths of supersymmetric particles into final states involving gravitinos.

The relevant interactions of the gravitino are given by [1, 15]

$$
\mathcal{L} \supset \frac{1}{8 M} \bar{\lambda}^{A} \gamma^{\rho} \sigma^{\mu \nu} \tilde{G}_{\rho} F_{\mu \nu}^{A}+\frac{1}{\sqrt{2} M} \bar{\psi}_{L} \gamma^{\mu} \gamma^{\nu} \tilde{G}_{\mu} D_{\nu} \phi+\text { h.c. }
$$

where the spin $3 / 2$ gravitino field is $\tilde{G}_{\mu}$ and $\lambda^{A}$ is the gaugino associated with the gauge field contained in the field strength $F_{\mu \nu}^{A}$, and $(\phi, \psi)$ are the scalar and fermionic components of the chiral supermultiplets. The full gravitino field can be well-approximated by its spin $1 / 2$ goldstino component when it appears as an external state in processes at energy scales relevant for collider studies:

$$
\tilde{G}_{\mu} \approx \sqrt{\frac{2}{3}} \frac{i}{m_{\tilde{G}}} \partial_{\mu} \tilde{G}
$$

In this limit it is not difficult to use eq. (5) to calculate decay rates of supersymmetric particles in the MSSM to final states including gravitinos. Let us first consider the decays of neutralinos. 
Using the relation between the mass eigenstates and the gauge eigenstates of the $\tilde{N}_{i}$, one finds the decay widths of $\tilde{N}_{i}$ into neutral gauge bosons to be as follows:

$$
\begin{aligned}
\Gamma\left(\tilde{N}_{i} \rightarrow \gamma \tilde{G}\right) & =\frac{\kappa_{i \gamma}}{48 \pi} \frac{m_{\tilde{N}_{i}}^{5}}{M^{2} m_{\tilde{G}}^{2}} \\
\Gamma\left(\tilde{N}_{i} \rightarrow Z \tilde{G}\right) & =\frac{2 \kappa_{i Z_{T}}+\kappa_{i Z_{L}}}{96 \pi} \frac{m_{\tilde{N}_{i}}^{5}}{M^{2} m_{\tilde{G}}^{2}}\left(1-\frac{m_{Z}^{2}}{m_{\tilde{N}_{i}}^{2}}\right)^{4}
\end{aligned}
$$

where

$$
\begin{aligned}
& \kappa_{i \gamma}=\left|N_{i 1} \cos \theta_{W}+N_{i 2} \sin \theta_{W}\right|^{2} \\
& \kappa_{i Z_{T}}=\left|N_{i 1} \sin \theta_{W}-N_{i 2} \cos \theta_{W}\right|^{2} \\
& \kappa_{i Z_{L}}=\left|N_{i 3} \cos \beta-N_{i 4} \sin \beta\right|^{2}
\end{aligned}
$$

measure the contents in $\tilde{N}_{i}$ of photino, zino, and the higgsino partner of the neutral would-be Nambu-Goldstone boson, respectively. (Here and in the following we use the notations of [16] for the parameters and mixing matrices of neutralinos and Higgs scalar bosons in the MSSM. Thus $N_{i j}$ are the neutralino mixing matrices with $(i, j)$ the (mass, gauge) eigenstate labels, and $\tan \beta$ is the ratio of Higgs vacuum expectation values.) In these and similar formulas below, the Planck-scale suppression $m_{\tilde{N}_{i}}^{2} / M^{2}$ is numerically counteracted by the hierarchy $m_{\tilde{N}_{i}}^{2} / m_{\tilde{G}_{\tilde{G}}}^{2}$, so that the decay width can be non-negligible. So for example we can write (7) in the suggestive form

$$
\Gamma\left(\tilde{N}_{i} \rightarrow \gamma \tilde{G}\right)=1.12 \times 10^{-11} \mathrm{GeV} \kappa_{i \gamma}\left(\frac{m_{\tilde{N}_{i}}}{100 \mathrm{GeV}}\right)^{5}\left(\frac{m_{\tilde{G}}}{1 \mathrm{eV}}\right)^{-2}
$$

When the 2-body decay $\tilde{N}_{i} \rightarrow Z \tilde{G}$ is near threshold, the formula (8) is not reliable; also when the 2-body decay is not kinematically allowed, the decay can still proceed through an off-shell $Z$ boson. In these situations one must use the 3-body decay formula. In the limit of massless SM fermions from the off-shell $Z$, the width of the neutralino from 3-body decays through a virtual $Z$ boson are obtained by replacing

$$
\left(2 \kappa_{i Z_{T}}+\kappa_{i Z_{L}}\right)\left(1-\frac{m_{Z}^{2}}{m_{\tilde{N}_{i}}^{2}}\right)^{4} \longrightarrow 2 \kappa_{i Z_{T}} I_{1}+\kappa_{i Z_{L}} I_{0}
$$

in (8), where the kinematic factors are most compactly written as

$$
I_{n}=\frac{\epsilon}{\pi} \int_{0}^{1} d x \frac{(1-x)^{4}(x / R)^{n}}{(x-R)^{2}+\epsilon^{2}}
$$

with $R=m_{Z}^{2} / m_{\tilde{N}_{i}}^{2}$ and $\epsilon=\Gamma_{Z} m_{Z} / m_{\tilde{N}_{i}}^{2}$. In the case that $m_{\tilde{N}_{i}}-m_{Z} \gg \Gamma_{Z}$, one finds $I_{0} \approx I_{1} \approx$ $\left(1-m_{Z}^{2} / m_{\tilde{N}_{i}}^{2}\right)^{4}$ so that $(8)$ is recovered. At threshold $\left(m_{\tilde{N}_{i}} \approx m_{Z}\right)$ one finds $I_{0} \approx 4 I_{1} \approx .0029$, 
rather than 0 as suggested by the 2-body formula (8). For another point of reference, with $m_{\tilde{N}_{1}}=70 \mathrm{GeV}$ one finds the kinematic factors are approximately $I_{0} \approx .0013$ and $I_{1} \approx .00015$. In order for off-shell or near-threshold decays $\tilde{N}_{1} \rightarrow Z^{(*)} \tilde{G}$ to compete with $\tilde{N}_{1} \rightarrow \gamma \tilde{G}$, the photino component of $\tilde{N}_{1}$ clearly would have to be very small. Even for $m_{\tilde{N}_{1}}=150 \mathrm{GeV}$ one finds $I_{0} \approx I_{1} \approx 0.16$.

Next we consider the decays of a neutralino into a gravitino plus any of the neutral Higgs scalar boson mass eigenstates $\varphi=\left(h^{0}, H^{0}, A^{0}\right)$ of the MSSM. The 2-body decay widths are given by

$$
\Gamma\left(\tilde{N}_{i} \rightarrow \varphi \tilde{G}\right)=\frac{\kappa_{i \varphi}}{96 \pi} \frac{m_{\tilde{N}_{i}}^{5}}{M^{2} m_{\tilde{G}}^{2}}\left(1-\frac{m_{\varphi}^{2}}{m_{\tilde{N}_{i}}^{2}}\right)^{4}
$$

where the relevant higgsino contents are given by

$$
\begin{aligned}
& \kappa_{i h^{0}}=\left|N_{i 3} \sin \alpha-N_{i 4} \cos \alpha\right|^{2} \\
& \kappa_{i H^{0}}=\left|N_{i 3} \cos \alpha+N_{i 4} \sin \alpha\right|^{2} \\
& \kappa_{i A^{0}}=\left|N_{i 3} \sin \beta+N_{i 4} \cos \beta\right|^{2}
\end{aligned}
$$

Note that with the identifications $\varphi=G^{0}$ (the electroweak would-be Nambu-Goldstone boson), $\kappa_{i G^{0}}=\left|N_{i 3} \cos \beta-N_{i 4} \sin \beta\right|^{2}$ and $m_{G^{0}}=m_{Z}$, one recovers the decay width into longitudinal $Z$ 's indicated in equation (8), in compliance with the equivalence theorem 17].

It is certainly not an outlandish possibility that $m_{\tilde{N}_{1}}>m_{h^{0}}$, so that the two body decay $\tilde{N}_{1} \rightarrow h^{0} \tilde{G}$ can compete with $\tilde{N}_{1} \rightarrow \gamma \tilde{G}$. However, this decay is also crippled by the kinematic suppression indicated in (15) unless $m_{\tilde{N}_{1}}$ is significantly larger than $m_{h^{0}}$. The 3-body decay widths for $\tilde{N}_{i} \rightarrow \varphi^{*} \tilde{G}$ with $\varphi^{*}$ decaying into pairs of SM fermions (treated as massless for kinematic purposes) are given by replacing in (15)

$$
\left(1-\frac{m_{\varphi}^{2}}{m_{\tilde{N}_{i}}^{2}}\right)^{4} \longrightarrow I_{1}
$$

as given by (14) with $R=m_{\varphi}^{2} / m_{\tilde{N}_{i}}^{2}$ and $\epsilon=\Gamma_{\varphi} m_{\varphi} / m_{\tilde{N}_{i}}^{2}$. Since in the MSSM the width of $h^{0}$ is only a few $\mathrm{MeV}$, such three-body and near-threshold decays are generally negligible.

The decay widths of sleptons and heavy squarks are also easily found. For a sfermion decaying into a massless SM fermion + gravitino, one finds the 2-body width

$$
\Gamma(\tilde{f} \rightarrow f \tilde{G})=\frac{m_{\tilde{f}}^{5}}{48 \pi M^{2} \tilde{m}_{\tilde{G}}^{2}}
$$

One of the more intriguing possibilities is that the nearly degenerate right-handed sleptons $\tilde{e}_{R}, \tilde{\mu}_{R}, \tilde{\tau}_{R}$ act effectively as co-NLSPs. In that case, the supersymmetry discovery signatures 
would generally involve at least two energetic leptons $+\mathbb{E}_{T}$. If a sneutrino is the NLSP, then signatures should often be similar to those in the neutralino LSP scenario, since the decays $\tilde{\nu} \rightarrow \nu \tilde{G}$ are invisible. It does not appear to be sensible to contemplate a left-handed charged slepton as the NLSP, because of the sum rule

$$
m_{\tilde{\ell}_{L}}^{2}=m_{\tilde{\nu}}^{2}+m_{W}^{2}|\cos 2 \beta|
$$

for $\tan \beta>1$. Squark (mass) ${ }^{2}$ parameters receive large positive contributions proportional to $\alpha_{s}^{2}$ and/or $\alpha_{s}$, so that it seems doubtful that a squark could be the NLSP. One possible exception is the lightest top squark mass eigenstate $\left(\tilde{t}_{1}\right)$ since $m_{\tilde{t}_{1}}^{2}$ can receive large negative radiative corrections proportional to the top Yukawa coupling squared. However, if $m_{\tilde{t}_{1}} \lesssim m_{t}$, then $\tilde{t}_{1}$ should be very long-lived if it is the NLSP, and in particular should always hadronize and escape the detector as a charge 0 or 1 "mesino" $\left(\tilde{t}_{1} \bar{q}\right)$ or as a charge 0,1 , or 2 "sbaryon" $\left(\tilde{t}_{1} q q^{\prime}\right)$ bound state. In any case, for the remainder of this paper, we will decline to consider the possibility that a sfermion could be the NLSP.

The 2-body decay widths of charginos $\left(\tilde{C}_{i}\right)$ into gravitino final states are given by formulas entirely analogous to (8) and (15):

$$
\begin{aligned}
\Gamma\left(\tilde{C}_{i}^{+} \rightarrow W^{+} \tilde{G}\right) & =\frac{2 \kappa_{i} W_{T}+\kappa_{i W_{L}}}{96 \pi} \frac{m_{\tilde{C}_{i}}^{5}}{M^{2} m_{\tilde{G}}^{2}}\left(1-\frac{m_{W}^{2}}{m_{\tilde{C}_{i}}^{2}}\right)^{4} \\
\Gamma\left(\tilde{C}_{i}^{+} \rightarrow H^{+} \tilde{G}\right) & =\frac{\kappa_{i H^{+}}}{96 \pi} \frac{m_{\tilde{C}_{i}}^{5}}{M^{2} m_{\tilde{G}}^{2}}\left(1-\frac{m_{H^{+}}^{2}}{m_{\tilde{C}_{i}}^{2}}\right)^{4}
\end{aligned}
$$

with

$$
\begin{aligned}
\kappa_{i W_{T}} & =\frac{1}{2}\left(\left|V_{i 1}\right|^{2}+\left|U_{i 1}\right|^{2}\right) \\
\kappa_{i W_{L}} & =\left|V_{i 2}\right|^{2} \sin ^{2} \beta+\left|U_{i 2}\right|^{2} \cos ^{2} \beta \\
\kappa_{i H^{+}} & =\left|V_{i 2}\right|^{2} \cos ^{2} \beta+\left|U_{i 2}\right|^{2} \sin ^{2} \beta
\end{aligned}
$$

The generalizations to off-shell decays are given by the obvious analogs of the above expressions for $\tilde{N}_{i}$ decays. However, it should be noted that because of the form of the chargino and neutralino mass matrices, a chargino can only be the NLSP in a small and not particularly attractive region of parameter space.

In general, if $m_{\tilde{G}}$ could be arbitrarily small compared to superpartner masses, then all decays of supersymmetric particles could proceed directly to the corresponding SM particle plus gravitino. However, as a practical matter for supersymmetric states accessible at Tevatron energies and taking into account a conservative lower bound on the gravitino mass as mentioned in the Introduction or stricter bounds in particular classes of models, it is easy to see that 
the decay widths for non-NLSP sparticles listed above should be quite small and should be overwhelmed by the usual well-studied decays. Besides the decay of the NLSP, there is one other potential exception which seems worthy of mention. If the gravitino mass is near the lower end of the allowed window, it is possible that a heavy gluino can decay directly to gluon+gravitino through a 2-body decay, rather than following the usual cascade decay pattern through virtual squarks. Since the only other decays of the gluino are mediated by virtual squarks which can be quite heavy in models, it is conceivable that the direct decay to gravitino can dominate in the gravitino LSP scenario. (In contrast, decays of all other non-NLSP superpartners can proceed through virtual $W \mathrm{~s}$ or $Z$ s or sparticles which are plausibly much lighter than squarks.) The relevant decay width is given by

$$
\Gamma(\tilde{g} \rightarrow g \tilde{G})=\frac{m_{\tilde{g}}^{5}}{48 \pi M^{2} m_{\tilde{G}}^{2}}=1.1 \times 10^{-9} \mathrm{GeV}\left(\frac{m_{\tilde{g}}}{250 \mathrm{GeV}}\right)^{5}\left(\frac{m_{\tilde{G}}}{1 \mathrm{eV}}\right)^{-2}
$$

The competition between this decay and the usual cascade decays of gluinos has already been studied in [18, 19], where (27) was found to be negligible unless $m_{\tilde{G}} \lesssim 10^{-2} \mathrm{eV}$, even if all squarks are as heavy as several $\mathrm{TeV}$. For gravitinos lighter than $10^{-2} \mathrm{eV}$, it was found that the direct decays (27) can dominate over the more conventional decay chains through virtual squarks only if

$$
m_{\tilde{G}} \lesssim 10^{-3} \mathrm{eV}\left(\frac{m_{\tilde{q}}}{1 \mathrm{TeV}}\right)^{2} .
$$

This can only occur in the slightly problematic case that $\Lambda_{\text {SUSY }}$ does not greatly exceed $m_{\tilde{q}}[\mathrm{cf}$. equation (11)].

Given the considerations above, we will optimistically assume for the remainder of this paper that a neutralino is the NLSP, that the branching fraction for $\tilde{N}_{1} \rightarrow \gamma \tilde{G}$ is $100 \%$, and that all supersymmetric decay chains terminate in this subdecay. Nevertheless, it is worth noting that this decay can be strongly suppressed due to a very small photino content of $\tilde{N}_{1}$ in regions of parameter space with small $|\mu|$, making $\tilde{N}_{1}$ long-lived on collider scales. Assuming the usual gaugino mass unification condition (四) and restricting our attention to the parameter space not already excluded by LEP with $\tan \beta>1.5, \kappa_{1 \gamma}$ can be less than 0.001 only if $\mu$ is negative and $|\mu| / M_{2}<0.2$. For $\kappa_{1 \gamma}<0.01$, it is required that $|\mu| / M_{2}<0.4(0.2)$ for $\mu$ negative (positive). A milder but still quite significant suppression $\kappa_{1 \gamma}<0.1$ can be obtained if $|\mu| / M_{2}<$ 0.5 (0.65) for $\mu$ negative (positive). Conversely, as long as $|\mu|>M_{2}$ and $m_{\tilde{C}_{1}}>50 \mathrm{GeV}$, one finds $\kappa_{1 \gamma}>0.21(0.13)$ for $\mu$ negative (positive). Formally, if $\kappa_{1 \gamma}$ were to vanish, the decay $\tilde{N}_{1} \rightarrow \gamma \tilde{G}$ could still proceed through one-loop graphs, but these amplitudes are very small [20] and in the present context are only competitive for a fine-tuning of $\kappa_{1 \gamma}$ which is finer than we are willing to contemplate here.

Of course, a $100 \%$ branching fraction for $\tilde{N}_{1} \rightarrow \gamma \tilde{G}$ does not guarantee that the photons 
can be detected, since the length scale associated with this decay might easily be comparable to the relevant physical size of the detector. The probability that each $\tilde{N}_{1}$ with energy $E$ in the lab frame will travel a distance $\leq x$ before decaying is given by

$$
P(x)=1-e^{-x / L}
$$

where from (7) the decay length is

$$
L=1.76 \times 10^{-3}\left(\kappa_{1 \gamma}\right)^{-1}\left(E^{2} / m_{\tilde{N}_{1}}^{2}-1\right)^{1 / 2}\left(\frac{m_{\tilde{N}_{1}}}{100 \mathrm{GeV}}\right)^{-5}\left(\frac{m_{\tilde{G}}}{1 \mathrm{eV}}\right)^{2} \mathrm{~cm} .
$$

Clearly $L$ depends strongly on $m_{\tilde{N}_{1}}$ and $m_{\tilde{G}}$ and can either be larger than, or negligible compared to, the relevant physical dimension $(\sim 150 \mathrm{~cm})$ of a CDF-type detector. Note that if $L$ is larger than $150 \mathrm{~cm}$, the efficiency for detecting one photon can greatly exceed that for detecting both. For example, taking $L$ to be 15 meters, one finds that the probability for both (one) of the photons being emitted within $150 \mathrm{~cm}$ of the event vertex is roughly $0.01(0.17)$. For $L \approx 150$ $\mathrm{cm}$, the probability of two (one) photons being emitted within $150 \mathrm{~cm}$ of the event vertex is 0.40 (0.47). Since the SM backgrounds for events with one energetic photon greatly exceed those for events with two such photons, we will optimistically assume in the following discussion that for the processes of interest $L<150 \mathrm{~cm}$, so that all supersymmetric events will lead to two potentially detectable photons. Taking $\kappa_{1 \gamma}$ and $\left(E^{2} / m_{\tilde{N}_{1}}^{2}-1\right)^{1 / 2}$ to be of order unity, this requires roughly $m_{\tilde{G}} \lesssim 250 \mathrm{eV}$ for a $100 \mathrm{GeV} \tilde{N}_{1}$. Larger decay lengths will decrease the efficiency of detection accordingly.

\section{Supersymmetry with a light gravitino at the Tevatron}

The presence of two energetic photons from supersymmetric events in the gravitino LSP scenario should dramatically increase the detectability over that found in the usual neutralino LSP scenario. In this section we will study the possibility for detecting inclusive $\gamma \gamma \mathbb{E}_{T}+$ $X$ signals at the Tevatron in the present data sample of about $100 \mathrm{pb}^{-1}$ per detector. We concentrate on signals from chargino and neutralino, slepton, and light stop squark production for a range of models, and we comment on other potential signals. For this study, we assume that the decays $\tilde{N}_{1} \rightarrow \gamma \tilde{G}$ occur within the detector $100 \%$ of the time. As a practical matter, we compute kinematics of events with the further assumption that these decays occur close to the event vertex. All event simulation is performed using the PYTHIA Monte Carlo with supersymmetric extensions [21]. Refs. [9, 10, 11] also contain recent studies of gravitino LSP physics at colliders. 


\section{A. Chargino and Neutralino Production}

The production cross sections for $\tilde{C}_{i} \tilde{C}_{j}, \tilde{C}_{i} \tilde{N}_{j}$ and $\tilde{N}_{i} \tilde{N}_{j}$ at hadron colliders are functions of the gaugino-higgsino parameters (the $U(1)$ gaugino mass $M_{1}$, the $S U(2)$ gaugino mass $M_{2}$, the supersymmetric Higgsino mass parameter $\mu, \tan \beta)$ and the squark masses. In the following we vary these parameters to find the range of expected signals. The gravitino LSP scenario has striking phenomenological implications for $\tilde{C}_{i}$ and $\tilde{N}_{i}$ production. Consider, for example, the process $p \bar{p} \rightarrow \tilde{C}_{1}^{ \pm} \tilde{N}_{2}$. In the neutralino LSP scenario, it is well-known that this process can be detected with a good efficiency when the final state includes three leptons [22]. However, in the gravitino LSP scenario, all of the final states of this process (including $\gamma \gamma \ell^{+} \ell^{-} \ell^{\prime+} \mathbb{E}_{T}$, $\gamma \gamma \ell^{+} j j \mathbb{E}_{T}, \gamma \gamma \ell^{+} \ell^{-} j j \mathbb{E}_{T}$ and $\left.\gamma \gamma j j j j \mathbb{E}_{T}\right)$ can provide useful signals. Likewise, the production of $\tilde{C}_{1}^{+} \tilde{C}_{1}^{-}$pairs can lead to observable signals $\gamma \gamma \ell^{+} \ell^{\prime-} \mathbb{E}_{T}, \gamma \gamma \ell^{ \pm} j j \mathbb{E}_{T}$, and $\gamma \gamma j j j j \mathbb{E}_{T}$. When the gaugino unification condition (4) is satisfied, these two processes provide the bulk of the supersymmetric signal throughout much of parameter space, because of the relatively large couplings $W \tilde{C}_{1} \tilde{N}_{2}$ and $Z \tilde{C}_{1} \tilde{C}_{1}, \gamma \tilde{C}_{1} \tilde{C}_{1}$. Also $\tilde{N}_{1} \tilde{N}_{1}$ production, which is undetectable at hadron colliders in the neutralino LSP scenario, leads to the signal $\gamma \gamma \mathbb{E}_{T}$ in the gravitino LSP scenario. Unfortunately, although this process is kinematically favored, it usually has a negligible cross section at hadron colliders because of a small $Z \tilde{N}_{1} \tilde{N}_{1}$ coupling and heavy squarks.

The branching fractions for the various final states associated with chargino and neutralino production are quite model-dependent. For example, the jet or lepton multiplicity and kinematics can be a strong function of squark and slepton masses. Since all of these final states from $\tilde{C}_{i}$ and $\tilde{N}_{i}$ production involve two energetic photons and missing transverse energy, we prefer to focus on the inclusive $\gamma \gamma \mathbb{E}_{T}+X$ signal rather than details of jet or lepton multiplicity. If a number of events are found in the data sample which are not understood as coming from the SM, then such details could help disentangle the underlying theory. Below we show such distributions for a specific set of models.

In Fig. 1 w we show the allowed range for the total inclusive production cross-section $\sigma(p \bar{p} \rightarrow$ $\tilde{N}_{i} \tilde{N}_{j}$ or $\tilde{C}_{i} \tilde{C}_{j}$ or $\left.\tilde{N}_{i} \tilde{C}_{j}\right)$ at $\sqrt{s}=1.8 \mathrm{TeV}$ as a function of the lightest neutralino mass $m_{\tilde{N}_{1}}$. We have assumed that the gaugino mass unification assumption (4) holds, so that the gauginohiggsino sector is determined by only three parameters, one of which we choose to be the $\tilde{N}_{1}$ mass. To generate this graph, we have varied the other parameters of the MSSM over the ranges

$$
\begin{gathered}
250 \mathrm{GeV}<m_{\tilde{q}}<1000 \mathrm{GeV} \\
-1000 \mathrm{GeV}<\mu<1000 \mathrm{GeV} \\
1.5<\tan \beta<55
\end{gathered}
$$




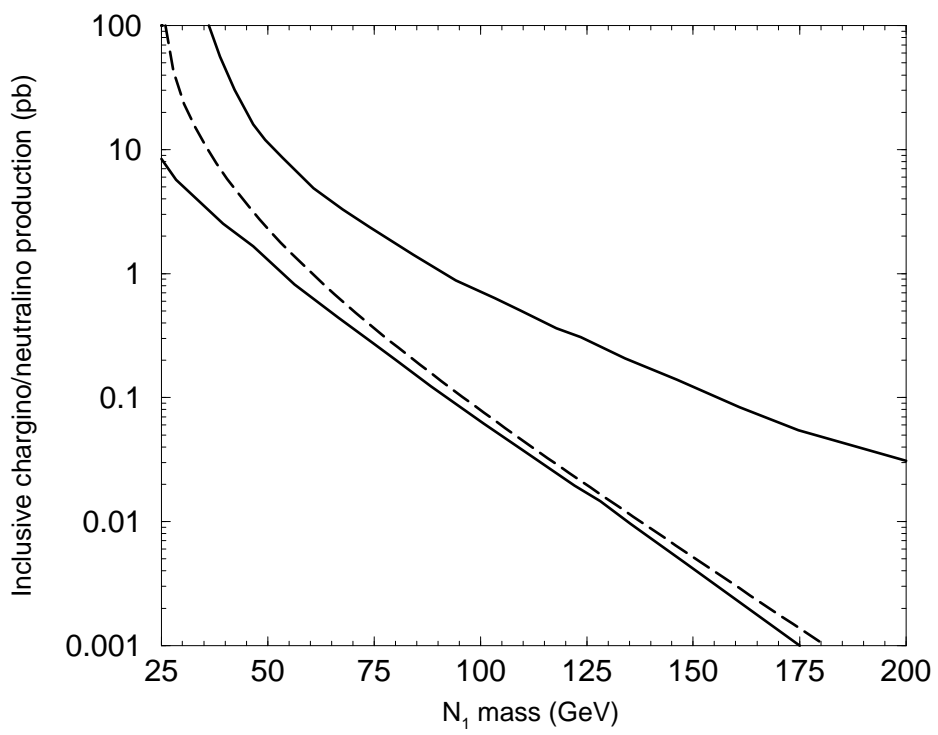

Figure 1: Total production cross sections for charginos and neutralinos $\left(\tilde{N}_{i} \tilde{N}_{j}\right.$ and $\tilde{N}_{i} \tilde{C}_{j}$ and $\tilde{C}_{i} \tilde{C}_{j}$ ) at the Tevatron with $\sqrt{s}=1.8 \mathrm{TeV}$ as a function of the lightest neutralino mass, assuming gaugino mass unification. The solid lines are the minimum and maximum allowed cross sections. The dashed line is a typical large $|\mu|$ and heavy squark limit $\left(\mu=1000 \mathrm{GeV}, m_{\tilde{q}}=1000 \mathrm{GeV}\right.$, $\tan \beta=1.5)$.

The dashed line represents a typical large $|\mu|$, heavy squark limit, namely $\mu=1000 \mathrm{GeV}$, $m_{\tilde{q}}=1000 \mathrm{GeV}, \tan \beta=1.5$. If the gaugino mass unification condition (蛋) is not satisfied, then the total inclusive chargino-neutralino production cross-section (for the range of $m_{\tilde{N}_{1}}$ shown) can be essentially negligible; this is traceable directly to the kinematic suppression associated with very heavy charginos. In Fig. 2 we show the same cross-section, this time as a function of the lighter chargino mass. Again, Fig. 目 assumes (4). However, we found that the minimum production cross-section in the case of general gaugino mass parameters is not significantly lower than that shown in Fig. 2, for a given $\tilde{C}_{1}$ mass. This is important because it shows that the Tevatron can set model-independent exclusion limits on $m_{\tilde{C}_{1}}$, if the efficiency for detection is reasonably bounded from below. The maximum production cross-section for general gaugino mass parameters not obeying (目) can be several times larger than that shown in Fig. 2. As can be seen from Fig. 2, the total number of chargino/neutralino pair production events at the Tevatron with $100 \mathrm{pb}^{-1}$ of data is $>100$ before cuts if a chargino mass is less than $100 \mathrm{GeV}$ (the maximum LEP reach).

To fully define a signal, we choose the following cuts for the two photons $\gamma_{1}, \gamma_{2}$ :

- $E_{T}\left(\gamma_{1}\right), E_{T}\left(\gamma_{2}\right)>12$ or $25 \mathrm{GeV}$, where $E_{T}$ is the transverse energy. We define two 


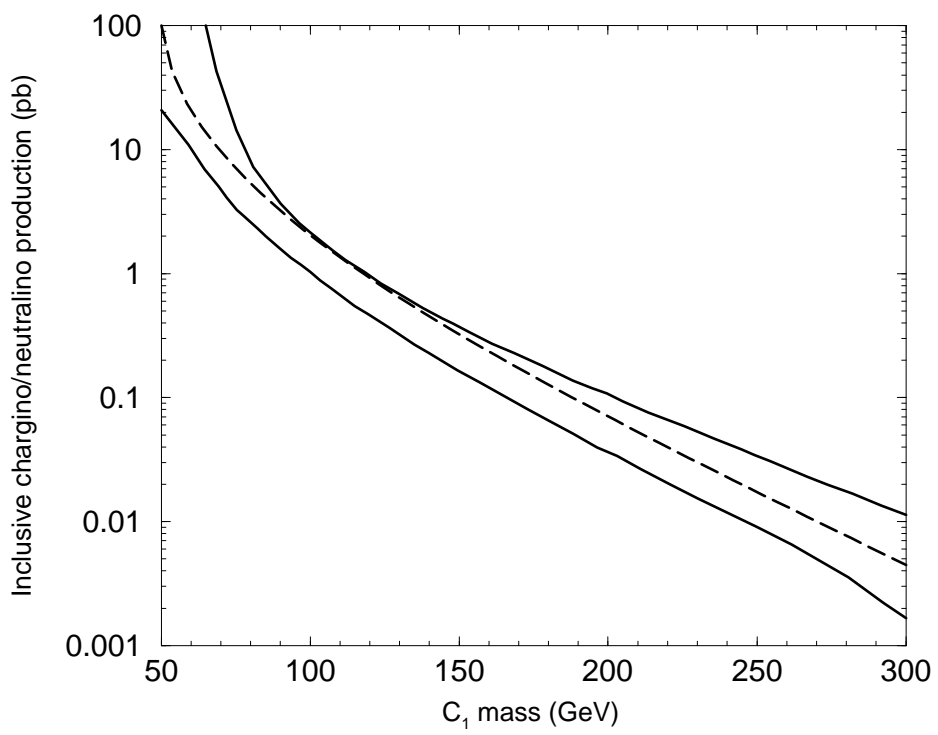

Figure 2: As in Fig. 1, but as a function of $m_{\tilde{C}_{1}}$. We find that even when the gaugino mass unification assumption (4) is not made, the minimum cross section is never significantly less than shown here.

different signals based on the minimal $E_{T}$. For the lower threshold, we impose a $30 \%$ loss of efficiency when one or both photons have $E_{T}$ below $25 \mathrm{GeV}$, to simulate the approximate loss of triggering efficiency [23].

- $\left|\eta\left(\gamma_{1}\right)\right|,\left|\eta\left(\gamma_{2}\right)\right|<1$, where $\eta$ is the pseudorapidity.

- $E_{T}^{\text {iso }}<4 \mathrm{GeV}$ 2], where

$$
E_{T}^{\text {iso }}=\sum_{j, R<0.4} E_{T}^{(j)}-E_{T}(\gamma)
$$

and we sum the transverse energy from all particles $(j)$ within a cone of size $R=\sqrt{(\Delta \eta)^{2}+(\Delta \phi)^{2}}$ centered on the photon candidate. (Photons from jets or bremsstrahlung tend not to be isolated from additional hadronic activity.)

- $\mathbb{E}_{T}>30 \mathrm{GeV}$, where $\mathbb{E}_{T}$ is determined by the sum of the visible energy in smeared jets, photons, and leptons.

Standard Model physics backgrounds can arise from $W^{ \pm}\left(\rightarrow \ell \nu_{\ell}\right) \gamma \gamma, Z\left(\rightarrow \nu \bar{\nu}, \tau^{+} \tau^{-}\right) \gamma \gamma$, and $Q \bar{Q} \gamma \gamma$ where $Q=c, b$, or $t$. We have not made a full matrix element simulation of these backgrounds. However, we estimate their magnitude by generating $W^{ \pm} \gamma, Z \gamma, t \bar{t}, b \gamma X$, and

\footnotetext{
${ }^{2}$ This number can be significantly reduced without affecting the signal.
} 
$c \gamma X$ events with additional QED radiation in the leading-logarithm approximation [24]. Based on this analysis, we estimate $\left(0.13,0.11,<10^{-3},<10^{-2},<0.1\right)$ events in $100 \mathrm{pb}^{-1}$ from each of these sources when $E_{T}(\gamma)>12 \mathrm{GeV}$. For this set of cuts, the $c \gamma \gamma$ background is the largest hadronic source, and it is well under control. Likewise, backgrounds from a jet faking an isolated photon can be estimated from these numbers using a simple scaling by $\alpha_{s} / \alpha_{e m} \times R_{j \rightarrow \gamma} \times f \approx .1$, where $R_{j \rightarrow \gamma} \approx 10^{-3}$ is the probability a jet fakes a photon and $f \approx 10$ accounts for the squared quark charge. The probability of two jets faking two photons is even further suppressed. Finally, backgrounds with a fake missing transverse energy are limited by excellent electromagnetic calorimetry. Essentially, these chosen cuts should yield a signal free from background, though the $\mathbb{E}_{T}$ cut could be increased if necessary.

To assess the sensitivity of the Tevatron to $\gamma \gamma \mathbb{E}_{T}+X$ signals from $\tilde{C}_{i}$ and $\tilde{N}_{i}$ production, we have performed event level simulations of various light gravitino models using the parameters:

$$
\begin{gathered}
M_{2}=100,150,200,225 \mathrm{GeV} \\
\mu= \pm 125, \pm 215, \pm 300, \pm 600 \mathrm{GeV} \\
\tan \beta=1.5,1.7,2.5,3.0,10.0 \\
\left(m_{\tilde{q}}, m_{\tilde{\ell}_{L}}, m_{\tilde{\ell}_{R}}\right)=(250,125,119) \text { or }(500,250,238) \mathrm{GeV} .
\end{gathered}
$$

Here $M_{1}$ is fixed by (41). The sneutrino mass is fixed by the sum rule (21). (When the result is less than the $\tilde{N}_{1}$ mass, we take instead $m_{\tilde{\nu}}=m_{\tilde{N}_{1}}+5 \mathrm{GeV}$ and $m_{\tilde{\ell}_{L}}$ fixed by the sum rule.) While larger squark masses can easily be obtained in models, we find that to a good approximation, the dependence of signals on squark mass vanishes for squark masses above $500 \mathrm{GeV}$ at the Tevatron. We display the results in terms of the number of expected events in $100 \mathrm{pb}^{-1}$ as a function of $\tilde{C}_{1}$ mass in Figs. 3 and 4 , for a minimum $E_{T}(\gamma)$ threshold of 12 and $25 \mathrm{GeV}$ respectively. The efficiency for detection of the signal is also displayed in Fig. 5 as a function of $m_{\tilde{N}_{1}}$ and in Fig. 6 as a function of $m_{\tilde{C}_{1}}$ using the $E_{T}(\gamma)>12 \mathrm{GeV}$ cut. By comparing Figs. 3 and 4 , we conclude that most of the photons originating from models with $m_{\tilde{C}_{1}} \geq 140 \mathrm{GeV}$ which pass the $12 \mathrm{GeV} E_{T}$ cut will also pass the higher threshold. It is also clear that the lower $E_{T}(\gamma)$ threshold substantially increases the signal for smaller $m_{\tilde{C}_{1}}$ despite the loss of triggering efficiency.

These figures suggest that the non-observation of a signal excludes $m_{\tilde{C}_{1}}<125 \mathrm{GeV}$ when (4) is assumed, which is well above the pair production threshold of any LEP upgrade. The same information, but plotted as a function of the $\tilde{N}_{1}$ mass, is shown in Fig. 17. From this plot, we conclude that a lightest neutralino mass below $70 \mathrm{GeV}$ is excludable in the same manner, when (4) is assumed. We have not attempted a completely general study of efficiencies when the gaugino mass unification is not assumed. However, we do not see any reason to expect significantly lower efficiencies in the completely general case. In particular, small mass differences between charginos and neutralinos should have little effect on the efficiency (for fixed $m_{\tilde{N}_{1}}$ ) since the photon energies and $\mathbb{E}_{T}$, which primarily determine the signal, depend on 


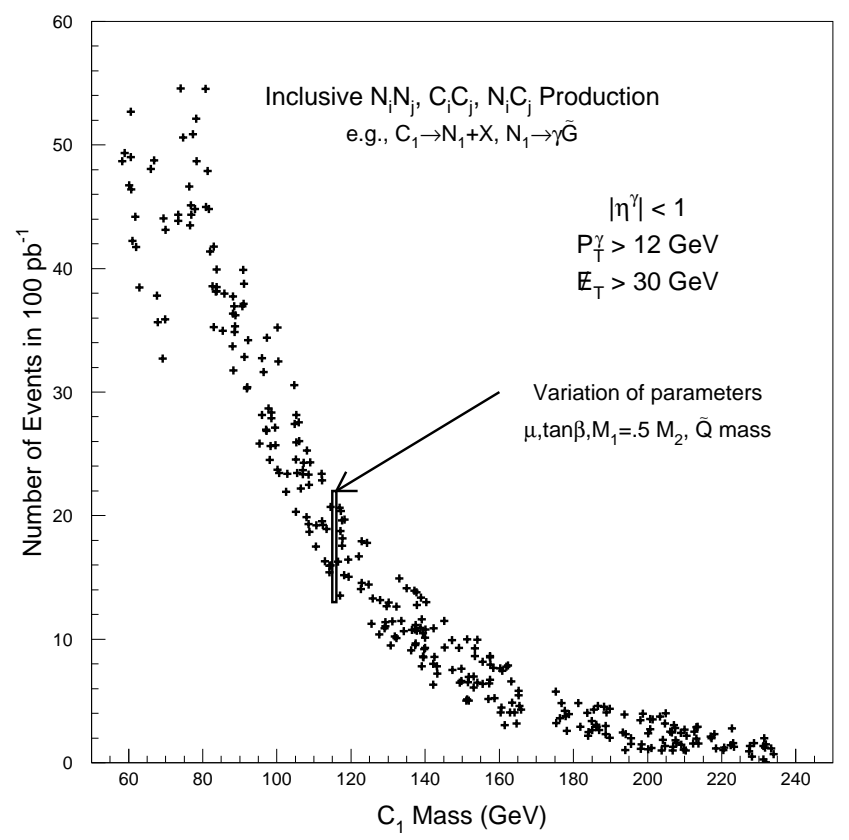

Figure 3: The number of $\gamma \gamma \mathbb{E}_{T}+X$ events expected in $100 \mathrm{pb}^{-1}$ of data using the models defined by (31) and the cuts explained in the text with $E_{T}(\gamma)>12 \mathrm{GeV}$. The signal comes from the inclusive production cross sections for charginos and neutralinos $\left(\tilde{N}_{i} \tilde{N}_{j}\right.$ and $\tilde{N}_{i} \tilde{C}_{j}$ and $\left.\tilde{C}_{i} \tilde{C}_{j}\right)$ at the Tevatron with $\sqrt{s}=1.8 \mathrm{TeV}$ as a function of the lightest chargino $\tilde{C}_{1}$ mass, assuming gaugino mass unification.

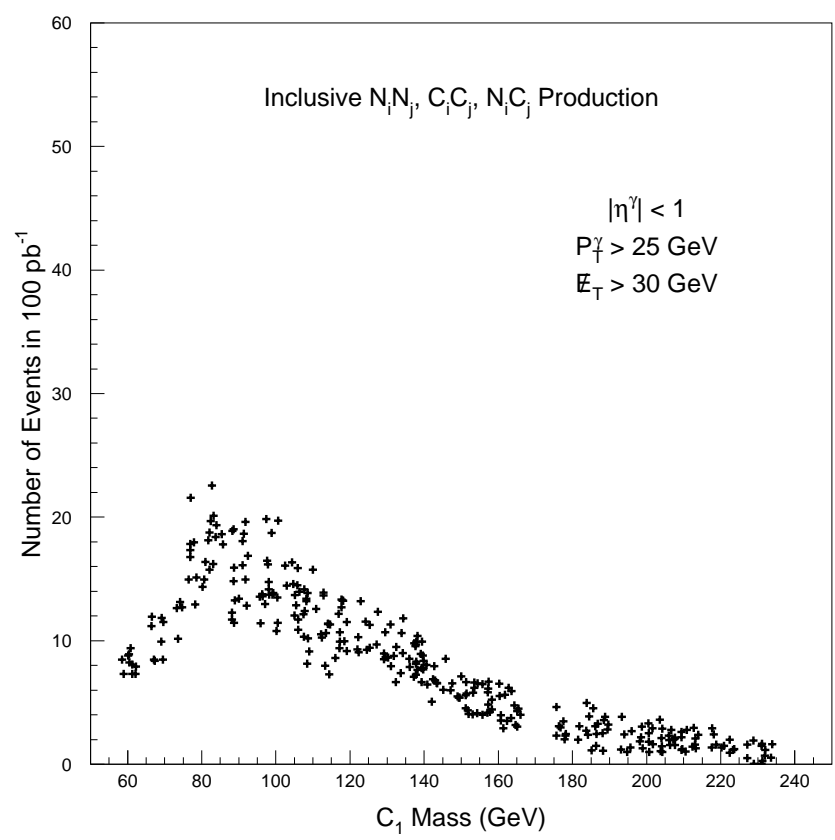

Figure 4: As in Fig. 3, but with $E_{T}(\gamma)>25 \mathrm{GeV}$. 


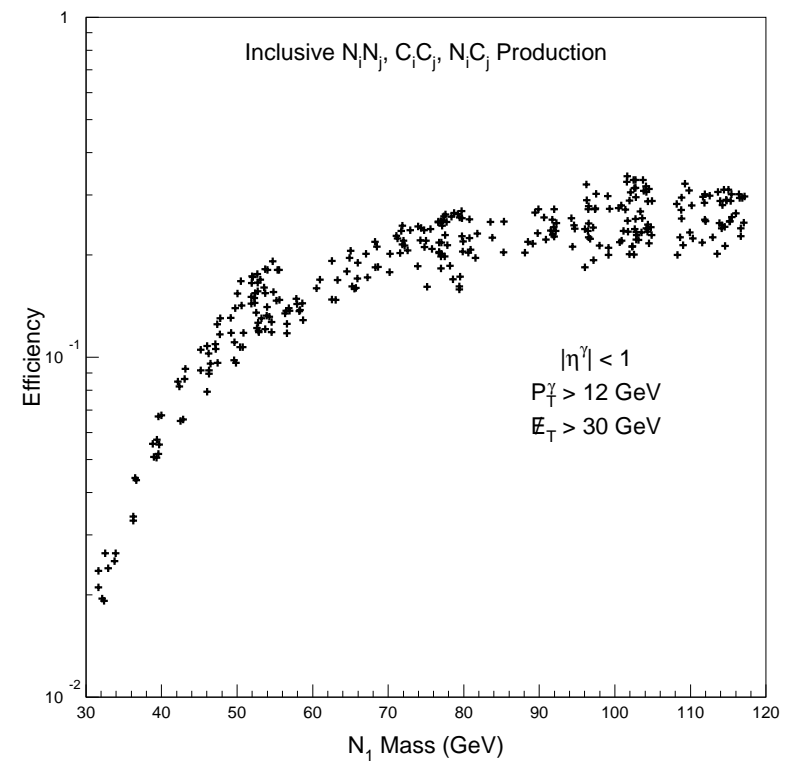

Figure 5: The number of generated $\gamma \gamma \mathbb{E}_{T}+X$ events passing cuts divided by the total for $E_{T}(\gamma)>12 \mathrm{GeV}$, for the models defined by (31). The signal comes from the inclusive production cross sections for charginos and neutralinos $\left(\tilde{N}_{i} \tilde{N}_{j}\right.$ and $\tilde{N}_{i} \tilde{C}_{j}$ and $\left.\tilde{C}_{i} \tilde{C}_{j}\right)$ at the Tevatron with $\sqrt{s}=1.8 \mathrm{TeV}$ as a function of the lightest chargino $\tilde{C}_{1}$ mass, assuming gaugino mass unification.

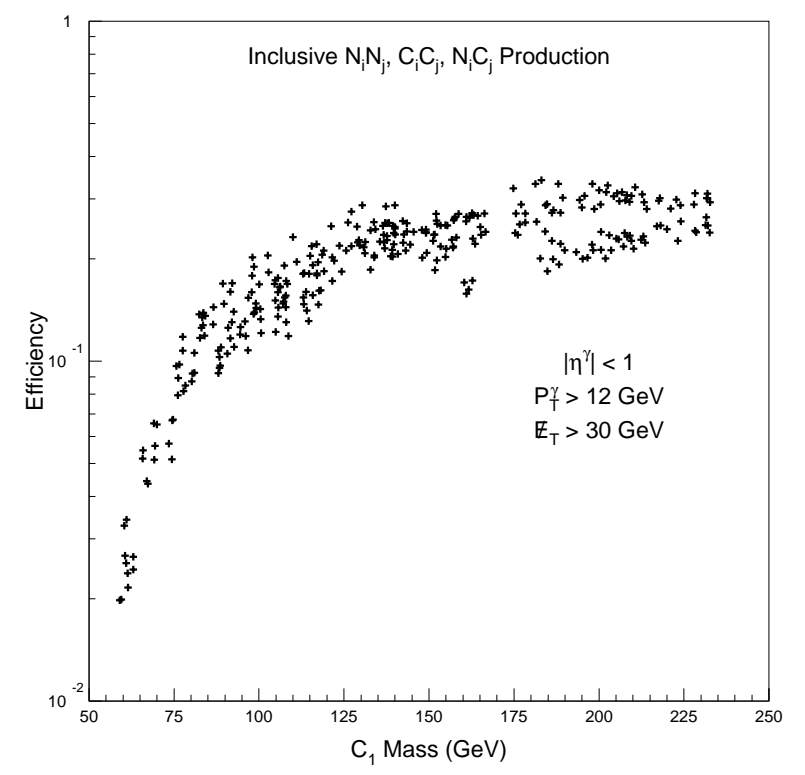

Figure 6: As in Figure 5, but as a function of $m_{\tilde{C}_{1}}$. 


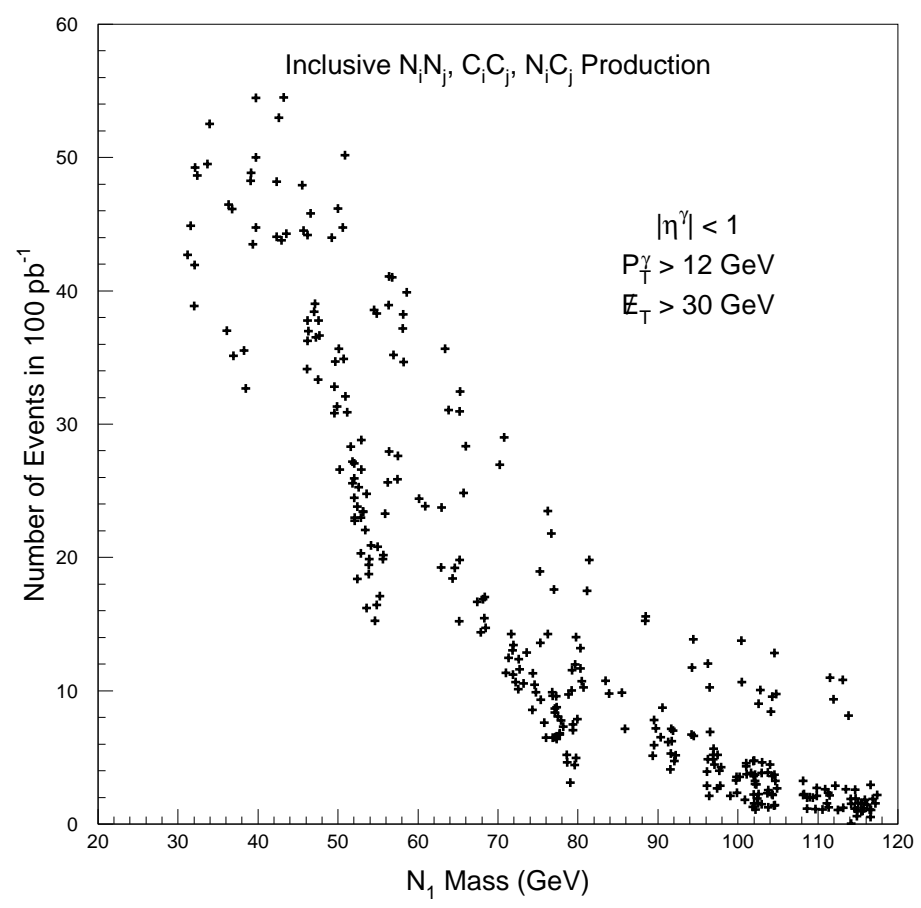

Figure 7: The number of $\gamma \gamma \mathbb{E}_{T}+X$ events expected in $100 \mathrm{pb}^{-1}$ of data using the cuts explained in the text with $E_{T}(\gamma)>12 \mathrm{GeV}$. The signal comes from the inclusive production cross sections for charginos and neutralinos $\left(\tilde{N}_{i} \tilde{N}_{j}\right.$ and $\tilde{N}_{i} \tilde{C}_{j}$ and $\left.\tilde{C}_{i} \tilde{C}_{j}\right)$ at the Tevatron with $\sqrt{s}=1.8 \mathrm{TeV}$ as a function of the lightest neutralino $\tilde{N}_{1}$ mass, assuming gaugino mass unification, and using the models defined by (31).

the mass and boost of $\tilde{N}_{1}$. Therefore by considering Figs. 5 and 6 and using the fact that the minimum production cross-section as a function of $m_{\tilde{C}_{1}}$ is bounded from below as in Fig. 2, we conclude that it should be possible to exclude $m_{\tilde{C}_{1}}<100 \mathrm{GeV}$ for $m_{\tilde{N}_{1}}>50 \mathrm{GeV}$ using the present $100 \mathrm{pb}^{-1}$ of Tevatron data, even without assuming (4).

As mentioned previously, the lepton and jet multiplicities of such events can be large, although they can be sharply reduced from naive expectations because of limited detector acceptance, jet definition, and isolation criteria. This is particularly relevant when the mass splittings among charginos and neutralinos are relatively small. Jets $(j)$ are defined using a standard clustering algorithm with $R=0.5$ and $E_{T}^{j}>15 \mathrm{GeV},\left|\eta^{j}\right|<2.5$. The particle energies are smeared using typical CDF energy resolutions. Electrons and muons must have $E_{T}^{(e, \mu)}>20$ $\mathrm{GeV}$ and $\left|\eta^{(e, \mu)}\right|<2.0$, while being isolated from excess transverse energy. We illustrate typical jet and lepton multiplicities for four specific models in Fig. 8. The model parameters are: 


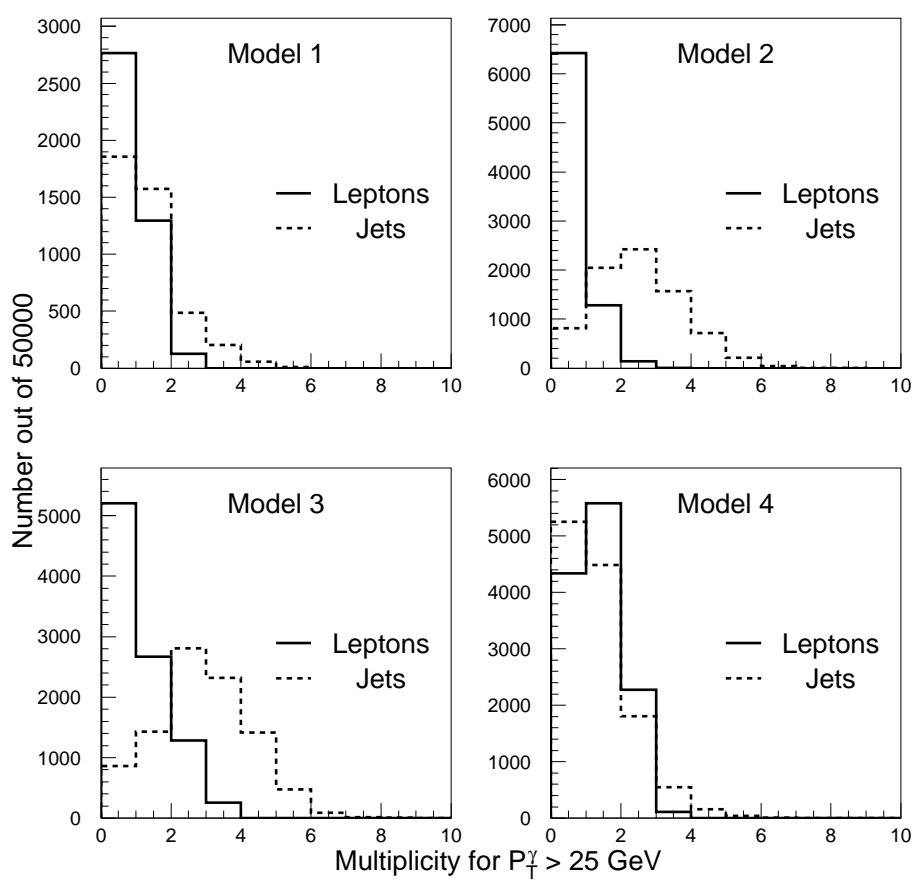

Figure 8: The lepton and jet multiplicities for the four models explained in the text.

- Model 1: $M_{2}=100 \mathrm{GeV}, \mu=-216 \mathrm{GeV}, \tan \beta=2.5, m_{\tilde{q}}=m_{\tilde{\ell}}=1000 \mathrm{GeV}$. One then finds $m_{\tilde{N}_{i}}=(53,108,227,238) \mathrm{GeV}, m_{\tilde{C}_{i}}=(108,240) \mathrm{GeV}, \kappa_{1 \gamma}=0.85$.

- Model 2: $M_{2}=150 \mathrm{GeV}, \mu=-125 \mathrm{GeV}, \tan \beta=1.7, m_{\tilde{q}}=m_{\tilde{\ell}}=1000 \mathrm{GeV}$. One then finds $m_{\tilde{N}_{i}}=(80,119,153,179) \mathrm{GeV}, m_{\tilde{C}_{i}}=(134,182) \mathrm{GeV}, \kappa_{1 \gamma}=0.81$.

- Model 3: $M_{2}=200 \mathrm{GeV}, \mu=600 \mathrm{GeV}, \tan \beta=3.0, m_{\tilde{\ell}_{R}}=150 \mathrm{GeV}, m_{\tilde{\nu}}=250$ $\mathrm{GeV}, m_{\tilde{\ell}_{L}}=260 \mathrm{GeV}, m_{\tilde{q}}=700 \mathrm{GeV}$. One then finds $m_{\tilde{N}_{i}}=(98,190,602,615) \mathrm{GeV}$ and $m_{\tilde{C}_{i}}=(189,614) \mathrm{GeV}$ and $\kappa_{1 \gamma}=0.72$.

- Model 4: $M_{2}=225 \mathrm{GeV}, \mu=300 \mathrm{GeV}, \tan \beta=1.5, m_{\tilde{\ell}_{R}}=105 \mathrm{GeV}, m_{\tilde{\nu}}=115$ $\mathrm{GeV}, m_{\tilde{\ell}_{L}}=125 \mathrm{GeV}, m_{\tilde{q}}=300 \mathrm{GeV}$. One then finds $m_{\tilde{N}_{i}}=(101,183,301,355) \mathrm{GeV}$ and $m_{\tilde{C}_{i}}=(176,350) \mathrm{GeV}$ and $\kappa_{1 \gamma}=0.56$.

We note in passing that Model 4 may be of particular interest, since it has some general properties consistent with an alternative candidate for the CDF ee $\gamma \gamma \mathbb{E}_{T}$ event through $\tilde{C}_{1} \tilde{C}_{1}$ production. In this model, the lepton multiplicity is peaked at 1 but there is also a substantial component with lepton multiplicity 2 (because of allowed 2-body decays of $\tilde{C}_{1}$ and $\tilde{N}_{2}$ to slepton $\left.+\tilde{N}_{1}\right)$. One expects about three chargino/neutralino events after cuts from this model in the 


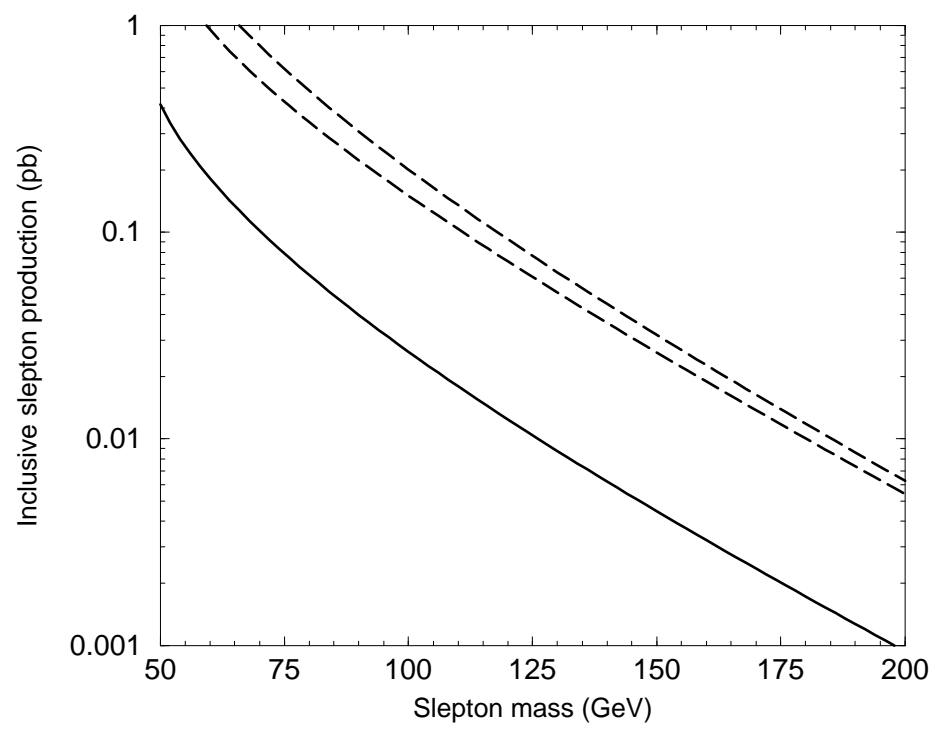

Figure 9: Total slepton production cross sections at the Tevatron with $\sqrt{s}=1.8 \mathrm{TeV}$, for $\tilde{\ell}_{R} \tilde{\ell}_{R}$ as a function of $m_{\tilde{\ell}_{R}}$ (solid line), and left-handed sleptons ( $\tilde{\nu} \tilde{\nu}$ and $\tilde{\ell}_{L} \tilde{\nu}$ and $\tilde{\ell}_{L} \tilde{\ell}_{L}$ ) as a function of $m_{\tilde{\nu}}$ (dashed lines). In the latter case, the lower (upper) dashed line corresponds to $\tan \beta=55$ (1.5). The cross sections shown are summed over slepton flavors, with slepton masses taken to be flavor-independent.

current data taken at the Tevatron. We will remark further on the chargino pair production interpretation of the CDF event below. This model also has light sleptons, so it could have produced the event through selectron pair production, but the kinematics do not favor this interpretation because the leptons would be too soft. Fig. 8 shows how the relative multiplicities could help distinguish models if a signal is established.

\section{B. Sleptons}

In most theoretical models, scalar (mass) ${ }^{2}$ parameters receive positive contributions proportional to $\alpha_{i}^{2}$ and/or $\alpha_{i}$, where $\alpha_{i}(i=1,2,3)$ are the gauge couplings felt by the scalar. Therefore one expects that sleptons with the same $S U(2)_{L} \times U(1)_{Y}$ quantum numbers should be degenerate in mass, and should all be considerably lighter than squarks, with $m_{\tilde{\ell}_{R}}<m_{\tilde{\nu}}<m_{\tilde{\ell}_{L}}$ the most plausible mass ordering. It is therefore interesting to consider slepton discovery signals at the Tevatron; a corresponding study in the neutralino LSP scenario appears in [25]. In Fig. 9, we show total Tevatron cross-sections for $\tilde{\ell}_{R} \tilde{\ell}_{R}$ production summed over three families (solid line) as a function of $m_{\tilde{\ell}_{R}}$. The signal for $\tilde{e}_{R} \tilde{e}_{R}$ production with the decay $\tilde{e}_{R} \rightarrow e \tilde{N}_{1}$ 


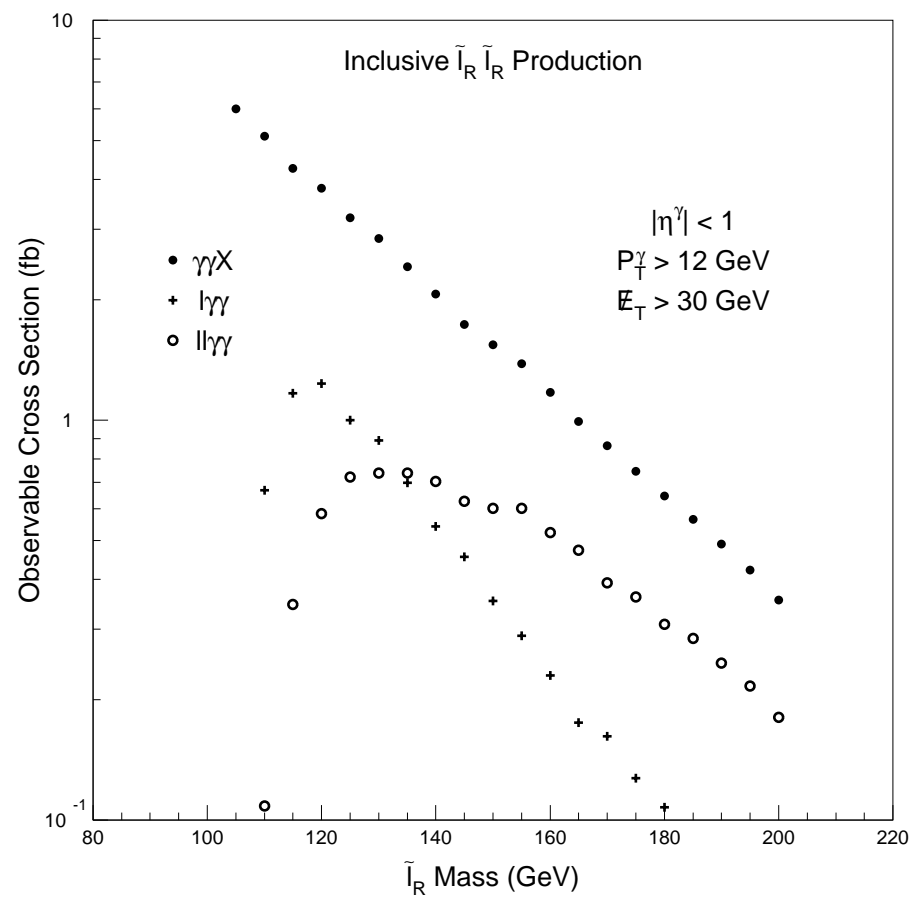

Figure 10: Expected $\tilde{\ell}_{R} \tilde{\ell}_{R}$ signals (including all three lepton flavors) from Model 4 at the Tevatron with $\sqrt{s}=1.8 \mathrm{TeV}$, as a function of $m_{\tilde{\ell}_{R}}$. The total $\gamma \gamma \mathbb{E}_{T}+X$ signal is shown by the filled circles, while the single lepton and dilepton components are denoted by crosses and open circles, respectively.

is ee $\gamma \gamma \mathbb{E}_{T}$, providing a viable candidate for the single observed CDF event of this type 12. As is known from the analyses of [12, 13, 14, such events do not seem to have a probable SM interpretation. In the same figure we show as a function of $m_{\tilde{\nu}}$ the total cross-section for $\tilde{\nu} \tilde{\nu}$, $\tilde{\ell}_{L} \tilde{\nu}$, and $\tilde{\ell}_{L} \tilde{\ell}_{L}$ production, for $\tan \beta=1.5$ and 55 (dashed lines). Since the masses of $\tilde{\nu}$ and $\tilde{\ell}_{L}$ are related by the sum rule (21), the rates for $\tilde{\ell}_{L} \tilde{\nu}$ and $\tilde{\ell}_{L} \tilde{\ell}_{L}$ production decrease monotonically with larger $\tan \beta$ (for a fixed value of $m_{\tilde{\nu}}$ ). The $\tilde{\ell}_{L} \tilde{\nu}$ component of the signal is always the largest. The final states from $\tilde{\ell}_{L} \tilde{\ell}_{L}$ and $\tilde{\ell}_{L} \tilde{\nu}$ production will depend specifically on the slepton and $\tilde{C}_{i}, \tilde{N}_{i}$ masses, but can contain $\gamma \gamma \mathbb{E}_{T}$ and up to three charged leptons.

Rather than conduct an extensive survey of slepton signatures, we consider as a test case the chargino/neutralino sector of Model 4 of subsection A. For the fixed set of gaugino parameters of that model, we further vary the right-handed selectron and sneutrino masses over the ranges $100 \mathrm{GeV}<m_{\tilde{\ell}_{R}}, m_{\tilde{\nu}}<200 \mathrm{GeV}$. It should be noted that for much of this range, direct decays of slepton to lepton and $\tilde{N}_{1}$ should dominate for the chosen model. Since the signals from righthanded sleptons and from left-handed sleptons have rather different characteristics, and because 


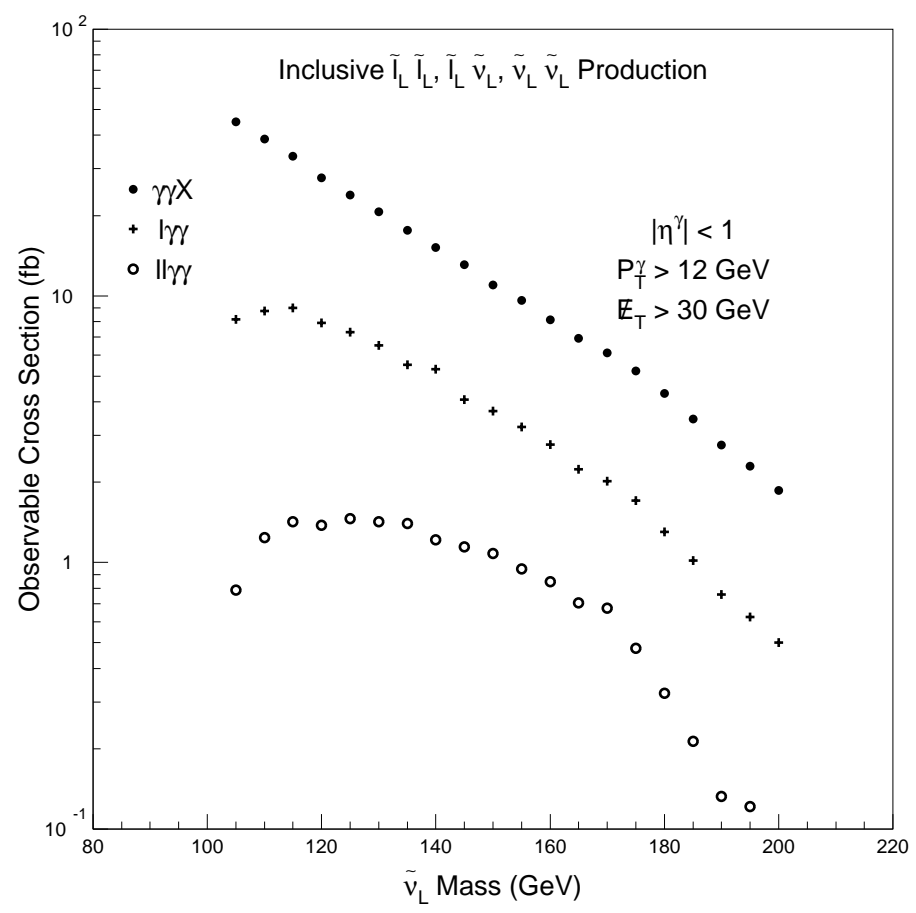

Figure 11: Total expected $\tilde{\ell}_{L} \tilde{\ell}_{L}$ and $\tilde{\ell}_{L} \tilde{\nu}$ and $\tilde{\nu} \tilde{\nu}$ signals (including all three lepton flavors) from Model 4 at the Tevatron with $\sqrt{s}=1.8 \mathrm{TeV}$, as a function of $m_{\tilde{\nu}}$. The total $\gamma \gamma \mathbb{E}_{T}+X$ signal is shown by the filled circles, while the single lepton and dilepton components are denoted by crosses and open circles, respectively.

the masses of right- and left-handed sleptons are a priori unrelated, we choose to display the results separately. In Fig. 10 we show the cross-sections after cuts arising from right-handed slepton production. The inclusive $\gamma \gamma \mathbb{E}_{T}$ signal, with or without additional leptons, is denoted by filled circles. We note that the dilepton component of the signal (open circles) is greater than the single lepton component (crosses) for $m_{\tilde{\ell}_{R}}>130 \mathrm{GeV}$. There is also a significant component with no leptons passing the cuts. In Fig. 11 we likewise show the total rate after cuts expected from $\tilde{\ell}_{L} \tilde{\ell}_{L}$ and $\tilde{\ell}_{L} \tilde{\nu}$, and $\tilde{\nu} \tilde{\nu}$ production, as a function of $m_{\tilde{\nu}}$. For any given model, the expected number of events with zero or one lepton far exceeds the number with two leptons. This is partly because of the comparatively larger cross-section for $\tilde{\nu}_{L}$ and $\tilde{\nu} \tilde{\nu}$ production, but also because some leptons do not pass cuts.

\section{Light stop squarks}

In specific models, one stop squark mass eigenstate $\left(\tilde{t}_{1}\right)$ is often found to be much lighter 


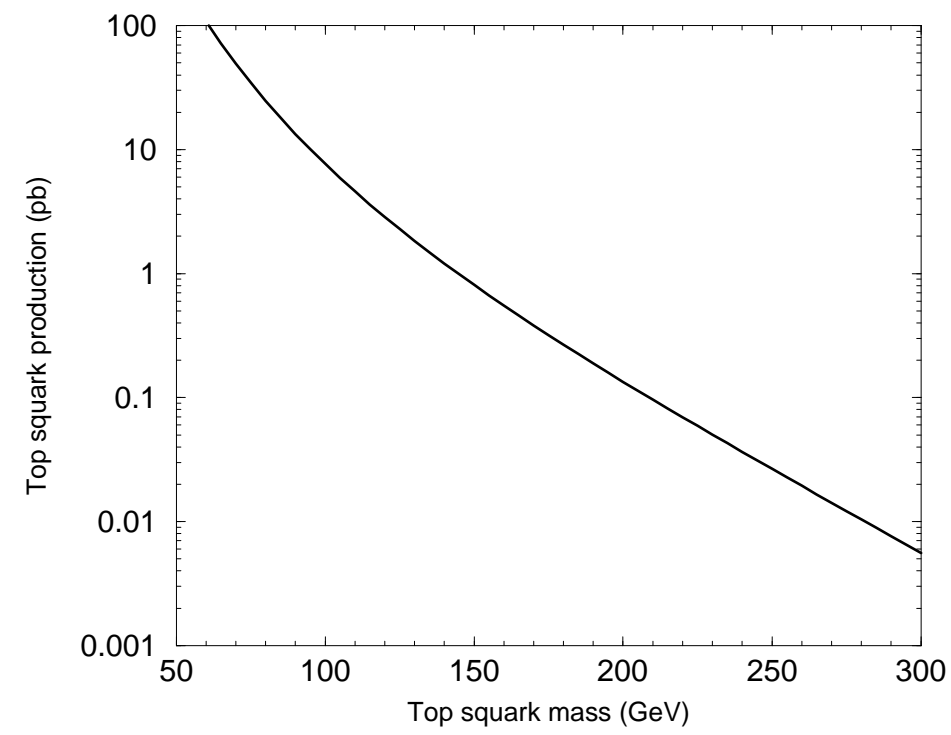

Figure 12: Cross section for pair-production of the lighter top squark mass eigenstate at the Tevatron with $\sqrt{s}=1.8 \mathrm{TeV}$.

than all of the other squarks, and can even be lighter than the top quark. If $m_{\tilde{t}_{1}}<100 \mathrm{GeV}$, chargino/stop loops might help to explain [26] the excess of $R_{b}$ in the LEP data. However, we have already seen that in the gravitino LSP scenario, charginos must be far too heavy for this to occur. Furthermore, a significant bound can be independently placed on a light stop squark mass in the gravitino LSP scenario given the integrated luminosity already obtained at the Tevatron. In Fig. 12, we show the total $\tilde{t}_{1} \tilde{t}_{1}^{*}$ pair production cross-section as a function of $\tilde{t}_{1}$ mass. In the gravitino LSP scenario, this process should lead to spectacular signals $\gamma \gamma \mathbb{E}_{T}+$ jets.

We consider two scenarios, based on the mass orderings $m_{\tilde{t}_{1}} \leq m_{\tilde{C}_{1}}+m_{b}$ or $m_{\tilde{t}_{1}}>m_{\tilde{C}_{1}}+m_{b}$. In the first case, each $\tilde{t}_{1}$ cascades through two two-body decays to a $c \gamma \tilde{G}$ final state. In the second, $\tilde{t}_{1}$ undergoes an additional three-body decay to reach a $b f \bar{f} \gamma \tilde{G}$ final state, where $f$ is a fermion. As a result, the photons produced in the second case tend to be softer. For the first case, $\tilde{t}_{1} \tilde{t}_{1}^{*}$ production leads to two additional charm jets in the final state, while $b$-jets and additional leptons or jets are present for the second. As before, we ignore such particulars, which could substantiate a suspected signal, and concentrate on the same inclusive $\gamma \gamma \mathbb{E}_{T}+X$ signal. Based on the previous bounds on $m_{\tilde{N}_{1}}$ and $m_{\tilde{C}_{1}}$, we consider models with $m_{\tilde{C}_{1}}+m_{b}>m_{\tilde{t}_{1}}>$ $m_{\tilde{N}_{1}}>70 \mathrm{GeV}$ for the range of gaugino parameters discussed previously, and a smaller set of models with $m_{\tilde{t}_{1}}>m_{\tilde{C}_{1}}+m_{b}>125 \mathrm{GeV}$. The results are illustrated in Fig. 13, which shows the number of expected diphoton events in the present data sample using the previously defined cuts with $E_{T}(\gamma)>12 \mathrm{GeV}$. There is a substantially higher detectability of the signal when 


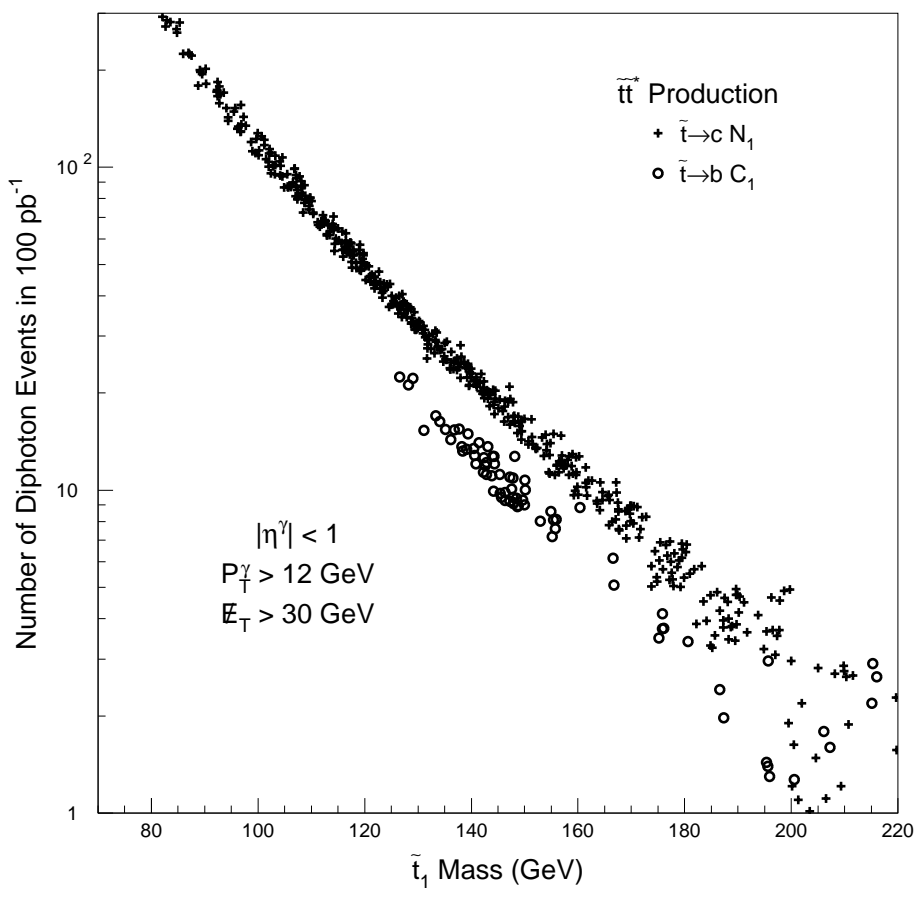

Figure 13: Number of $\gamma \gamma \mathbb{E}_{T}+X$ events in $100 \mathrm{pb}^{-1}$ as a function of the lighter top squark mass eigenstate at the Tevatron with $\sqrt{s}=1.8 \mathrm{TeV}$.

$\tilde{t}_{1} \rightarrow \tilde{C}_{1} b$ is not kinematically allowed. The absence of such events in the present data sample seems to exclude a $\tilde{t}_{1}$ lighter than at least $140 \mathrm{GeV}$ even in the case that $m_{\tilde{t}_{1}}>m_{\tilde{C}_{1}}+m_{b}$, which is already far too heavy to have any effect on the interpretation of the LEP $R_{b}$ measurement. Additionally, if $m_{\tilde{t}_{1}}+m_{\tilde{N}_{i}}<m_{t}$, then the decay $t \rightarrow \tilde{t}_{1} \tilde{N}_{i}$ can occur, generating $\gamma \gamma \mathbb{E}_{T}$ events from $t \bar{t}$ production, but the bounds on $m_{\tilde{t}_{1}}$ and $m_{\tilde{N}_{1}}$ preclude this.

Note that the limit on the mass of the light stop in the gravitino LSP scenario is much stronger than for the neutralino LSP scenario in the case where $\tilde{t}_{1} \rightarrow c \tilde{N}_{1}$. The latter case, which relies on the signal of two acollinear jets and $\mathbb{E}_{T}$, is limited by the mass splitting between $\tilde{t}_{1}$ and $\tilde{N}_{1}$ which determines the jet $E_{T}$ spectrum [27]. The main limitation of the gravitino LSP scenario is $m_{\tilde{N}_{1}}$ which sets both the scale of $E_{T}(\gamma)$ and $\mathbb{E}_{T}$.

\section{Other Processes}

It is a common feature of known models that the gluino and squarks are quite heavy. However, the presence of energetic photons in the eventual decay products means that the 


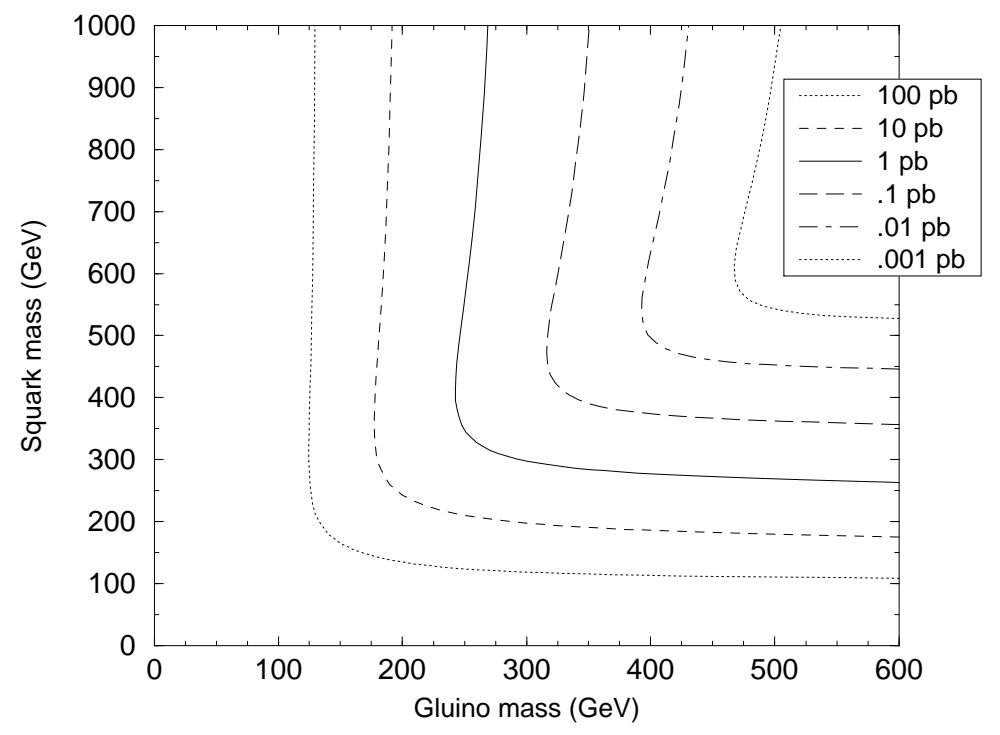

Figure 14: Contours of inclusive production cross sections for gluinos and squarks ( $\tilde{g} \tilde{g}$ and $\tilde{g} \tilde{q}, \tilde{q} \tilde{q})$ at the Tevatron with $\sqrt{s}=2 \mathrm{TeV}$. All squark flavors are taken to be degenerate for simplicity.

detection efficiency is likely to be higher in the gravitino LSP scenario than in the neutralino LSP scenario. Therefore it is again interesting to get an idea of the upper limit on the potential reach of the Tevatron collider by considering the total inclusive production of gluinos and squarks $(\tilde{g} \tilde{g}, \tilde{g} \tilde{q}, \tilde{q} \tilde{q})$. It must be mentioned that there are at least two factors which might adversely affect the efficiency here somewhat, including larger boosts leading to longer decay lengths for $\tilde{N}_{1} \rightarrow \gamma \tilde{G}$ [cf. eq. (30)], and losses from photon isolation requirements with the higher jet multiplicity. In Fig. 14, we show contours of this total production cross section, in the $\left(m_{\tilde{g}}, m_{\tilde{q}}\right)$ plane. (For simplicity, we have assumed degeneracy of all squark flavors.) At least in the case when gaugino mass unification is assumed, it appears to be doubtful that gluino pair-production processes can be useful, since the non-observation of events from chargino and neutralino production together with (何) forces the gluino mass to be too large. It should be noted that $M_{3}$ as given by (4) is less than the physical pole mass of the gluino [28] by an amount which is often quite substantial, especially if the squarks are heavier. This effect makes it even less likely that processes involving gluino production can compete with the chargino/neutralino processes already discussed. The rate for associated production of $\tilde{C}_{i}$ or $\tilde{N}_{i}$ with a gluino or squark overtakes the $(\tilde{g} \tilde{g}+\tilde{g} \tilde{q}+\tilde{q} \tilde{q})$ production rate when $m_{\tilde{g}}$ exceeds roughly $400 \mathrm{GeV}$, depending on the squark masses [29]. In this regime, however, even the sum of all processes involving gluino or squark production should be small compared to those from chargino and neutralino production, unless $m_{\tilde{q}}$ is significantly less than $m_{\tilde{g}}$. It therefore seems unlikely that 
gluinos or squarks can be involved in the discovery process.

\section{E. Comments on the interpretation of the CDF ee $\gamma \gamma \mathbb{E}_{T}$ event}

In this section we have emphasized the power of the Tevatron in setting exclusion limits in the gravitino LSP scenario. Of course, in this context we must mention that at least one event 112 of this general type has been observed at CDF. This event has an energetic electron and positron, two energetic photons each with $|\eta|<1$, and large $(>50 \mathrm{GeV}) \mathbb{E}_{T}$. It easily passes the cuts defining our signal above.

The most obvious candidate process for this event is selectron pair production. As has already been discussed in [13, 14], and recently in some more detail in [11], one can attempt to explain the event either in terms of $\tilde{e}_{R} \tilde{e}_{R}$ pair production or $\tilde{e}_{L} \tilde{e}_{L}$ pair production. From the kinematic information, we found in [14] that in either of these two cases, one has rough bounds $m_{\tilde{e}}>80 \mathrm{GeV}$ and $38<m_{\tilde{N}_{1}}<100 \mathrm{GeV}$. If one assumes gaugino mass unification, the lower bound obtained here for $m_{\tilde{N}_{1}}$ is far weaker than the lower bound established above from nonobservation of chargino and neutralino events at the Tevatron. As has been emphasized recently in [11], the energetic electrons in the event seem to indicate a significant mass difference between $m_{\tilde{N}_{1}}$ and $m_{\tilde{e}}$, in order to have sufficiently energetic electrons with a high enough probability to explain the event.

Right-handed selectrons have a lower production cross-section than do left-handed selectrons for a given mass, as can be seen from Fig. 9, and this seems to perhaps favor the idea that the pair-produced selectron was left-handed. However, since we are forced to calculate the probability of this single event using Poisson statistics, this argument is not on very solid footing. For example, if the three right-handed sleptons are degenerate in mass, then the crosssection to produce any pair $\tilde{\ell}_{R} \tilde{\ell}_{R}$ is of course 3 times larger than the rate for the pair $\tilde{e}_{R} \tilde{e}_{R}$ which could explain the event actually seen. It is not completely clear which of these rates should be taken in assessing the likelihood of a single observed event. In any case, the rate before cuts for $\tilde{\ell}_{R} \tilde{\ell}_{R}$ production in $100 \mathrm{pb}^{-1}$ is about 1 event for $m_{\tilde{\ell}_{R}}=125 \mathrm{GeV}$ and $1 / 2$ event for $m_{\tilde{\ell}_{R}}=145 \mathrm{GeV}$. The rates after cuts are significantly less because of acceptances (see Fig. 10), but it seems possible that the observed event is an upward fluctuation in the $\tilde{e}_{R} \tilde{e}_{R}$ production process, even with $m_{\tilde{e}_{R}}-m_{\tilde{N}_{1}}$ sufficiently large to explain the observed kinematics.

Conversely, although the rates for left-handed selectron production are larger, one must also note that in this interpretation the expected number of events with two leptons is always

considerably less than for one or zero leptons (see Fig. 11). The reason for this is that the production cross-section from $\tilde{\nu} \tilde{\nu}$ and $\tilde{\nu} \tilde{\ell}_{L}$ is necessarily larger than for $\tilde{\ell}_{L} \tilde{\ell}_{L}$ because of the 
kinematics dictated by the sum rule (21). It might therefore be viewed as problematic that the single observed event has two leptons. The limited acceptance for leptons only exacerbates this problem. Nevertheless, it again seems to be not entirely out of the question that the event could be due to $\tilde{e}_{L} \tilde{e}_{L}$ production.

There is another very interesting possibility, however, illustrated by model 4 above, that the event could be due to chargino pair-production. We note that depending on parameters, the chargino pair-production cross section remains sufficiently large to give 1 event (after cuts) in $100 \mathrm{pb}^{-1}$ up to at least $m_{\tilde{C}_{1}}=200 \mathrm{GeV}$. Now, each produced chargino can decay into either $\ell \nu \gamma \tilde{G}$ or $q \bar{q} \gamma \tilde{G}$. If the decay is dominantly through $W$-boson or squark exchange, then one might expect the ee $\gamma \gamma \mathbb{E}_{T}$ event to be accompanied by many more events with jets or significant hadronic activity in addition to $\gamma \gamma \mathbb{E}_{T}$. However, this can be avoided if $\tilde{C}_{1}$ has kinematically allowed 2-body decays into left-handed sleptons and not into $W \tilde{N}_{1}$ :

$$
\begin{aligned}
& m_{\tilde{C}_{1}}>m_{\tilde{\nu}}>m_{\tilde{N}_{1}} \\
& m_{\tilde{C}_{1}}-m_{\tilde{N}_{1}}<m_{W} .
\end{aligned}
$$

In this case, the chargino should decay as either $\tilde{C}_{1} \rightarrow \tilde{\nu} \ell \rightarrow \ell \nu \tilde{N}_{1} \rightarrow \ell \gamma \mathbb{E}_{T}$ or (if kinematically allowed) $\tilde{C}_{1} \rightarrow \tilde{\ell}_{L} \nu \rightarrow \ell \nu \tilde{N}_{1} \rightarrow \ell \gamma \mathbb{E}_{T}$. Both of these decays have the same signal (with different kinematics), so that the signal for chargino pair production will be $\ell^{+} \ell^{\prime-} \gamma \gamma \mathbb{E}_{T}$ in this case, with a nearly $100 \%$ branching fraction before cuts. Even though sneutrinos are lighter than charginos, the chargino production cross-section can be much larger. Of course, it should be noted that the rate for different flavor leptons in this case is twice that for like-flavor leptons. Still, we find that it is possible to obtain kinematics and rates after cuts which could explain the CDF event. The kinematics of the event together with the cross-section for chargino pairproduction evidently favor $m_{\tilde{C}_{1}} \gtrsim 125 \mathrm{GeV}$ in this case.

We should also mention that $\tilde{N}_{1} \tilde{N}_{2}$ production can give an ee $\gamma \gamma \mathbb{E}_{T}$ signal, but it is very difficult to reconcile this possibility with the observed event, because of the large invariant mass of the ee pair in the event [12].

\section{Supersymmetry with a light gravitino at LEP}

The LEP collider at CERN will probe some of the parameter space which is not yet excludable by the current $100 \mathrm{pb}^{-1}$ data sample collected at the Tevatron. However, it is important to take into account the results of Section 3 when assessing the discovery potential of the various LEP upgrades. At least within the context of gaugino mass unification, we have found that the lightest neutralino mass can be bounded from below by $70 \mathrm{GeV}$, based on 
the exclusion capability of the current $100 \mathrm{pb}^{-1}$ data. Similarly, the lighter chargino mass is bounded below by $125 \mathrm{GeV}$, and even when (函) is not assumed, one has $m_{\tilde{C}_{1}}>100 \mathrm{GeV}$ for $m_{\tilde{N}_{1}}>50 \mathrm{GeV}$. Therefore, it is immediately clear that one cannot hope to observe chargino pair production at any of the LEP upgrades considered here in the gravitino LSP scenario with our assumption that $\tilde{N}_{1} \rightarrow \tilde{G} \gamma$ always occurs within the detector. Furthermore, the second lightest neutralino should also not be kinematically accessible at LEP even in $\tilde{N}_{1} \tilde{N}_{2}$ production, at least in the case that gaugino mass unification (四) holds. The reason for this is that $m_{\tilde{C}_{1}}>125$ (as required by the Tevatron data) and $m_{\tilde{N}_{1}}<95$ (as required for accessibility in $e^{+} e^{-}$collisions with $\sqrt{s}=190 \mathrm{GeV}$ ) forces one into a region of parameter space with rather large $|\mu|$ and gaugino-like $\tilde{N}_{1}$ and $\tilde{N}_{2}$, so that $m_{\tilde{N}_{1}}+m_{\tilde{N}_{2}}>210 \mathrm{GeV}$. Therefore, it is clear that in the chargino/neutralino sector, LEP190 can only hope to observe $\tilde{N}_{1} \tilde{N}_{1}$ production with signature $\gamma \gamma \notin$. Likewise, the existing Tevatron data makes it impossible for a light stop (or other squark) to be accessible at LEP with our assumptions. There is a still a possibility to observe slepton pair production since, taking into account efficiencies, the Tevatron cannot set exclusion limits on slepton masses which are significantly stronger than the indirect one following from $m_{\tilde{\ell}}>m_{\tilde{N}_{1}}>70 \mathrm{GeV}$. Therefore there is a narrow range of $\tilde{N}_{1}$ and slepton masses from no less than $70 \mathrm{GeV}$ up to less than $95 \mathrm{GeV}$ which can be probed at LEP with $\sqrt{s} \leq 190 \mathrm{GeV}$.

We begin by considering $\tilde{N}_{1} \tilde{N}_{1}$ production in $e^{+} e^{-}$collisions, which leads to events with two acoplanar photons and large missing energy. (A similar study for the NLC has recently been made [9].) The energy distribution of photons produced[ in such events is flat, with endpoints

$$
\begin{aligned}
& E_{\min }<E_{\gamma_{1}}, E_{\gamma_{2}}<E_{\max } \\
& E_{\max , \min }=\frac{1}{4}\left(\sqrt{s} \pm \sqrt{s-4 m_{\tilde{N}_{1}}^{2}}\right) .
\end{aligned}
$$

The two photon energies in each event vary over this range independently, providing a very simple characteristic kinematic signature. The missing energy in each event is bounded according to $2 E_{\min }<\notin<2 E_{\max }$ and is peaked at $E_{\text {beam }} \equiv \sqrt{s} / 2$. Two further corollaries are that the distribution of $E_{\gamma_{1}}+E_{\gamma_{2}}$ is the same as that of $\not E$, and that the energy distribution of the more (less) energetic photon observed in each event rises (falls) linearly with energy. The numerical bounds on photon energies in the $\tilde{N}_{1} \tilde{N}_{1}$ signal are, for the various LEP upgrades:

$$
\begin{array}{ll}
20 \mathrm{GeV}<E_{\gamma_{1}}, E_{\gamma_{2}}<60 \mathrm{GeV} & (\sqrt{s}=160 \mathrm{GeV}) \\
18 \mathrm{GeV}<E_{\gamma_{1}}, E_{\gamma_{2}}<70 \mathrm{GeV} & (\sqrt{s}=175 \mathrm{GeV}) \\
16 \mathrm{GeV}<E_{\gamma_{1}}, E_{\gamma_{2}}<80 \mathrm{GeV} & (\sqrt{s}=190 \mathrm{GeV})
\end{array}
$$

for a lower bound $m_{\tilde{N}_{1}}=70 \mathrm{GeV}$. For masses nearer threshold, the range of photon energies of course becomes narrower around $\sqrt{s} / 4$ in each case. Thus the lower bound on $\tilde{N}_{1}$ mass from

\footnotetext{
${ }^{3}$ We neglect final state interference effects throughout the following discussion.
} 
the Tevatron ensures that the $\tilde{N}_{1} \tilde{N}_{1}$ signal at LEP will automatically pass appropriate cuts on soft photons. This will be useful below in our discussion of cuts and backgrounds.

Several factors affect the production cross-section for $\tilde{N}_{1} \tilde{N}_{1}$ at LEP. Since in the accessible parameter space $\tilde{N}_{1}$ is essentially forced to have a large gaugino component, the s-channel $Z$ boson exchange contribution is suppressed. If sleptons are light, the diagrams with slepton exchange will dominate. The diagrams with $\tilde{e}_{R}$ exchange are usually far more important, because the $e \tilde{e}_{R} \tilde{N}_{1}$ coupling is larger than the $e \tilde{e}_{L} \tilde{N}_{1}$ coupling. The $\tilde{N}_{1} \tilde{N}_{1}$ production crosssection is quite sensitive to the selectron masses, even if the selectrons themselves are not accessible at LEP. As a result, the discovery reach is always within a few $\mathrm{GeV}$ of the kinematic limit, but for no value of $m_{\tilde{N}_{1}}$ can one clearly guarantee discovery at any of the LEP upgrades, because of low observable rates for large $m_{\tilde{e}_{R}}$. At LEP160, the cross-section is always less than $0.2 \mathrm{pb}$ for models (with gaugino mass unification) not excludable at the Tevatron, and is less than $0.1 \mathrm{pb}$ for $m_{\tilde{N}_{1}}>75 \mathrm{GeV}$. These are optimistic upper bounds, and the cross-sections for less favorable parameters can be much smaller. This leaves open only the possibility of perhaps a few events at LEP160 with $25 \mathrm{pb}^{-1}$ per experiment, for a narrow mass range, optimistically $70 \mathrm{GeV}<m_{\tilde{N}_{1}}<77 \mathrm{GeV}$. As we will remark below, there is also a non-trivial background for such events, so that an unambiguous discovery will require a certain amount of luck.

The prospects for discovery (or confirmation) are clearly much brighter at LEP190 with 500 $\mathrm{pb}^{-1}$ per experiment, both because of the kinematic reach and the greater luminosity. In Figure 15, we show a scatter plot of the total $\tilde{N}_{1} \tilde{N}_{1}$ cross-section at $\sqrt{s}=190 \mathrm{GeV}$. Each point on this plot corresponds to a set of model parameters which plausibly could have avoided detection at the Tevatron with the current integrated luminosity, based on the results of the previous section. To illustrate the dominance of the $\tilde{e}_{R}$ exchange diagrams, models with $m_{\tilde{e}_{R}}<175 \mathrm{GeV}$ are denoted by crosses, while those with $175 \mathrm{GeV}<m_{\tilde{e}_{R}}<500 \mathrm{GeV}$ are denoted by dots. (The CDF ee $\gamma \gamma \mathbb{E}_{T}$ event could perhaps be explained by $\tilde{e}_{R} \tilde{e}_{R}$ production in models of the former category.) The gaugino mass unification condition (41) is assumed here. We have taken into account initial state radiation effects which imply a small ( $\lesssim 10 \%$ ) reduction in the signal; it should be noted that such effects are larger when $Z$ boson exchange dominates because of radiative return to the $Z$ peak. To these cross-sections one must apply detector cuts, e.g. [30]:

$$
\begin{aligned}
& \left|\cos \theta_{\gamma}\right|<0.95 \\
& \left(p_{T}\right)_{\gamma}>0.065 E_{\text {beam }},
\end{aligned}
$$

for each photon. The discovery reach with $500 \mathrm{pb}^{-1}$ extends up to within a few $\mathrm{GeV}$ of the kinematic limit. Clearly the presence of a light $\tilde{e}_{R}$ provides much more favorable discovery prospects. However, there is no guarantee of discovery of a light $\tilde{N}_{1}$ if $m_{\tilde{e}_{R}}$ is large, even with this amount of luminosity, and even for the most favorable kinematics. 


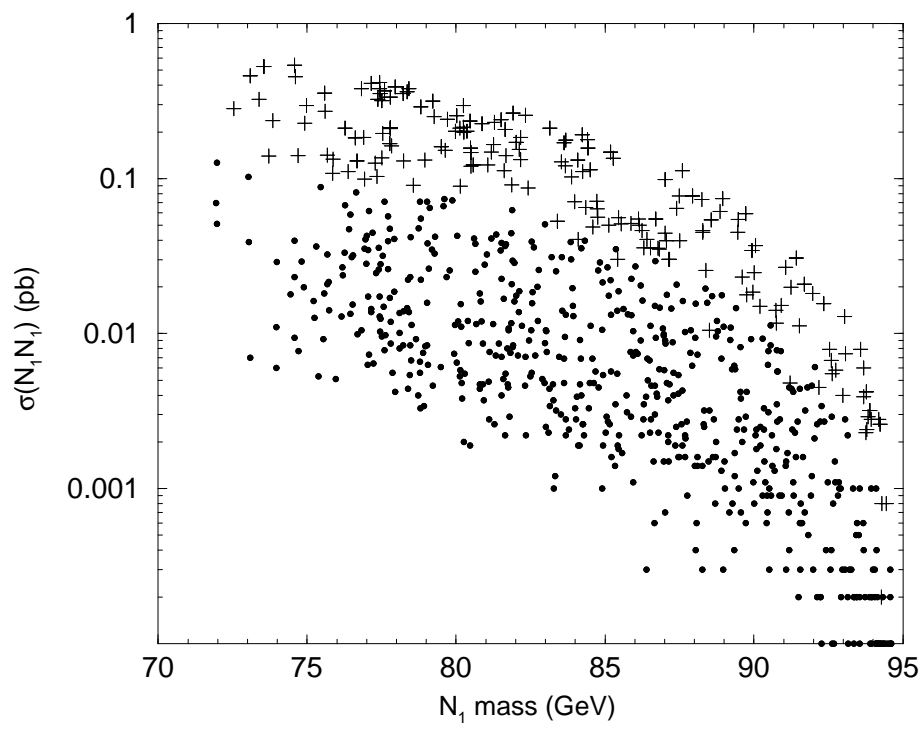

Figure 15: Production cross-section for $\tilde{N}_{1} \tilde{N}_{1}$ at LEP with $\sqrt{s}=190 \mathrm{GeV}$. Each point represents a set of model parameters which may not be excludable at the Tevatron with $100 \mathrm{pb}^{-1}$. The crosses (dots) represent models with $m_{\tilde{e}_{R}}$ less (greater) than $175 \mathrm{GeV}$.

We now turn to the question of backgrounds. The ordinary QED process $e^{+} e^{-} \rightarrow \gamma \gamma$ production has a large cross section, but can easily be discriminated against with a cut on missing energy or equivalently $E_{\gamma}<0.8 E_{\text {beam }}$ for each photon. The most important physics backgrounds for the $\gamma \gamma \notin$ signal come from $\gamma \gamma \nu_{i} \bar{\nu}_{i}(i=e, \mu, \tau)$ with two separately gaugeinvariant sets of diagrams:

A) $e^{+} e^{-} \rightarrow \gamma \gamma Z^{(*)}$ with $Z \rightarrow \nu_{i} \bar{\nu}_{i}$. (3 Feynman diagrams);

B) $e^{+} e^{-} \rightarrow \gamma \gamma \nu_{e} \bar{\nu}_{e}$ through virtual $W$-boson exchange. (7 Feynman diagrams).

We have computed these backgrounds using CompHEP [31], a specialized package for automated calculation of high-energy elementary particle processes, with results fed into BASES, a Monte Carlo phase-space integration program. The processes $e^{+} e^{-} \rightarrow \gamma \gamma \nu_{\mu} \bar{\nu}_{\mu}$ and $e^{+} e^{-} \rightarrow$ $\gamma \gamma \nu_{\tau} \bar{\nu}_{\tau}$ receive contributions only from the type A diagrams. At $\sqrt{s}=(160,175,190)$, they each contribute $(49,37,30)$ fb to the background for $\gamma \gamma \notin$ after the cuts (39), (40). When the final state is $\gamma \gamma \nu_{e} \bar{\nu}_{e}$, one must take into account a significant interference between the diagrams of types $\mathrm{A}$ and $\mathrm{B}$. The diagrams of type A clearly dominate in the kinematic regime characterized by a missing invariant mass $M_{\text {Invis }}$ very close to $M_{Z}$. [Here $M_{\text {Invis }}^{2}=\left(p_{e^{+}}+p_{e^{-}}-p_{\gamma_{1}}-p_{\gamma_{2}}\right)^{2}$.] In that regime, the interference with the type $\mathrm{B}$ diagrams are a small perturbation and in any case only affect $1 / 3$ of the background. For slightly larger $M_{\text {Invis }}$, however, the type B diagrams 
do have a substantial interference with the off-peak type A diagrams. The overall effect is one of constructive interference, but the sign is not definite for all kinematic configurations. At $\sqrt{s}=(160,175,190)$, we find $(61,49,42)$ fb for $e^{+} e^{-} \rightarrow \gamma \gamma \nu_{e} \bar{\nu}_{e}$ after the cuts (39), (40).

Since the $\gamma \gamma \nu \bar{\nu}$ backgrounds have larger support for relatively soft photon energies, one can reduce them somewhat by imposing the cut

$$
0.2<E_{\gamma} / E_{\text {beam }}<0.8
$$

on each photon; the upper limit easily eliminates the $e^{+} e^{-} \rightarrow \gamma \gamma$ process as we have already mentioned. The cut (41) has little or no effect on the signal, as can be seen from (34)-(38). After imposing this cut in addition to the detector cuts (39), (40), we find a remaining background at $\sqrt{s}=(160,175,190)$ of $(29,24,21)$ fb from $\gamma \gamma \nu_{e} \bar{\nu}_{e}$, and $(27,22,18)$ fb from each of $\gamma \gamma \nu_{\mu} \bar{\nu}_{\mu}$ and $\gamma \gamma \nu_{\tau} \bar{\nu}_{\tau}$.

In order to more strongly reduce the backgrounds we can impose a cut on the missing invariant mass of

$$
10 \mathrm{GeV}<M_{\text {Invis }}<80 \mathrm{GeV} \text {. }
$$

The upper limit is to avoid the $\gamma \gamma \nu \bar{\nu}$ physics backgrounds, while the lower limit eliminates a potentially large (several hundred fb) detector background following from $e^{+} e^{-} \rightarrow \gamma \gamma(\gamma)$ with one photon lost in the beam direction or in an insensitive part of the detector. (The part of this background due to photons lost in the beam direction is also substantially reduced by imposing a lower bound cut on $\not p_{T}$.) The signal vanishes at the endpoints of the distribution $M_{\text {Invis }}=0$, $2 E_{\max }$ and is broadly distributed in between. The greater part of the signal will always pass all of the cuts, although a significant part of the signal will necessarily have to be eliminated by the cut (42). After imposing this cut in addition to (39), (40), the total $\gamma \gamma \nu \bar{\nu}$ backgrounds at $\sqrt{s}=(160,175,190) \mathrm{GeV}$ are only $(1.8,1.3,1.0)$ fb respectively. Finally, imposing the cut (41) on top of these cuts reduces the background to a completely negligible level.

The distribution in $M_{\text {Invis }}$ for signals and backgrounds at $\sqrt{s}=190 \mathrm{GeV}$ are shown in Figure 16. In this figure we have arbitrarily chosen a total signal (before cuts) of $50 \mathrm{fb}$, with $\tilde{N}_{1}$ masses of 75 and $90 \mathrm{GeV}$. The differential cross-sections shown are after the detector cuts (39), (40) and photon energy cuts (41). The total $\gamma \gamma \nu \bar{\nu}$ background shown amounts to $56 \mathrm{fb}$, but is reduced to a negligible level by the $M_{\text {Invis }}$ cut. Note, however, the significant overlap in invariant missing mass for the backgrounds and the signals. The signal distribution in $M_{\text {Invis }}$ is broadly peaked below the $80 \mathrm{GeV}$ cut, and vanishes near $M_{\text {Invis }}=0$. We conclude that even in the worst-case kinematic situation, the efficiency for detecting $\tilde{N}_{1} \tilde{N}_{1}$ should exceed $50 \%$ after cuts at LEP190. Thus a $40 \mathrm{fb}$ signal before cuts should provide a 10 event discovery after cuts with $500 \mathrm{pb}^{-1}$. By comparing with Fig. 15, we conclude that LEP190 should be able to 


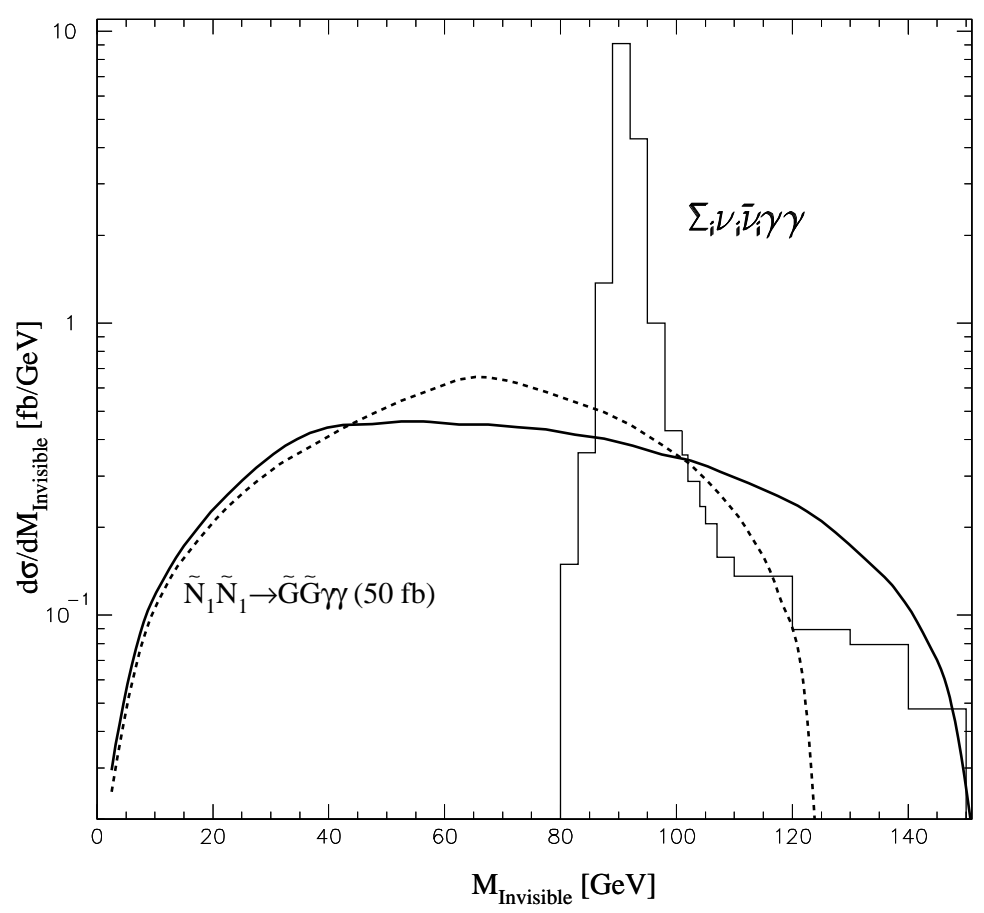

Figure 16: Distribution of the missing invariant mass in $\gamma \gamma \not \mathbb{E}$ events at LEP with $\sqrt{s}=190$ $\mathrm{GeV}$. Angular and photon energy cuts have been applied as described in the text. The lighter solid line is the remaining total background ( $56 \mathrm{fb}$ ) for all three neutrino species. The signals for $m_{\tilde{N}_{1}}=75$ and $90 \mathrm{GeV}$ are the solid and dashed lines, respectively, with an arbitrary choice of $50 \mathrm{fb}$ for the signal before cuts in each case.

unambiguously observe $\tilde{N}_{1} \tilde{N}_{1}$ production for $m_{\tilde{N}_{1}}$ up to at least $85 \mathrm{GeV}$ if $m_{\tilde{e}_{R}} \lesssim 175 \mathrm{GeV}$, assuming gaugino mass unification. The exclusion capability decreases for larger $m_{\tilde{e}_{R}}$, however. The $\gamma \gamma \nu \bar{\nu}$ background is more problematic at $\sqrt{s}=160 \mathrm{GeV}$ with $25 \mathrm{pb}^{-1}$ or $\sqrt{s}=175 \mathrm{GeV}$ with $10 \mathrm{pb}^{-1}$, where only at most a few signal events are expected, and the signal distribution in $M_{\text {Invis }}$ again overlaps with the $Z$-boson peak.

Conversely, Figure 17 shows the distributions for photon energies at $\sqrt{s}=190 \mathrm{GeV}$ for the backgrounds, before and after the $M_{\text {Invis }}$ cut. All of the $\gamma \gamma \nu \bar{\nu}$ contributions have been included here. The two distributions correspond to the more and less energetic photon in each background event, after the detector cuts (39), (40). After imposing in addition the cuts (41) and (42), the total $\gamma \gamma \nu \bar{\nu}$ background is reduced to a fraction of a femtobarn. The signal from $\tilde{N}_{1} \tilde{N}_{1}$ production (not shown) is characterized by a linearly rising (falling) distribution for the more (less) energetic photon in each event, with endpoints $E_{\min }$ and $E_{\max }$ as given above. (Note the $\log$ scale in Fig. 17.)

We now turn to the question of slepton pair production signals at LEP. In general, slep- 


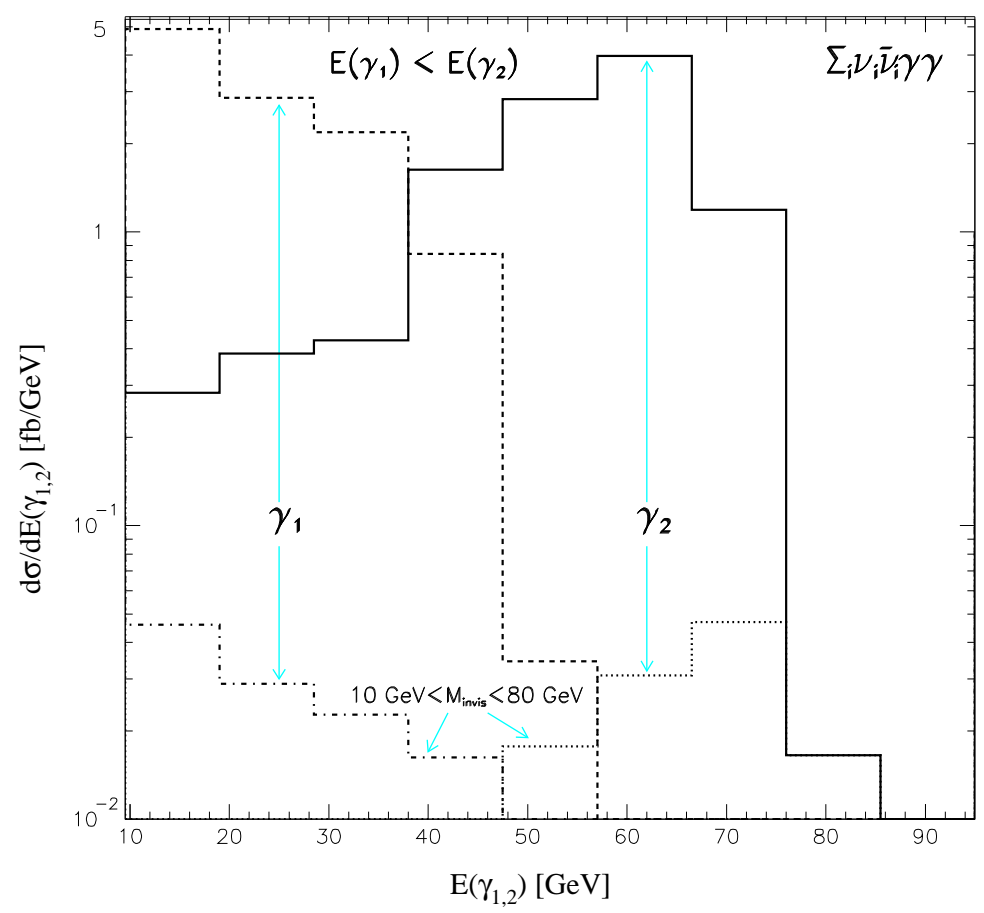

Figure 17: Distribution of photon energies for $\gamma \gamma \nu \bar{\nu}$ backgrounds at LEP with $\sqrt{s}=190$ $\mathrm{GeV}$. Detector cuts have been applied as described in the text. The solid (dashed) line is the distribution for the more (less) energetic photon $\gamma_{2}\left(\gamma_{1}\right)$ in each event. The dotted (dot-dash) lines are the same distributions after the cut on $M_{\text {Invis }}$ described in the text.

ton masses up to within a few $\mathrm{GeV}$ of the kinematic limit should lead to visible signals with $500 \mathrm{pb}^{-1}$ at LEP190. If right-handed sleptons are kinematically accessible, one finds that the cross-section for $\tilde{e}_{R} \tilde{e}_{R}$ production is generally somewhat larger than those for each of $\tilde{\mu}_{R} \tilde{\mu}_{R}$ and $\tilde{\tau}_{R} \tilde{\tau}_{R}$, because of the positive contribution of diagrams with t-channel exchange of gaugino-like $\tilde{N}_{1}$. The pair production of electron sneutrinos can be very strongly suppressed, because of destructive interference from chargino exchange, even with $\tilde{C}_{1}$ required to be heavier than 125 $\mathrm{GeV}$. Fortunately, pair production of muon and tau sneutrinos does not suffer this suppression, and those cross-sections are always large up to within a $\mathrm{GeV}$ or two of the kinematic limit. Because of the sum rule (21), it seems quite unlikely that pair production of left-handed selectrons can be a discovery process at LEP in the gravitino LSP scenario considered in this paper. However, the cross-section for $\tilde{e}_{L} \tilde{e}_{R}$ can be even larger than for $\tilde{e}_{R} \tilde{e}_{R}$ production when both are kinematically accessible, because of a large contribution from exchange of gaugino-like neutralinos.

It is important to note that for the (quite narrow) range of masses which are accessible at LEP and which cannot already be ruled out at the Tevatron, each slepton has one (and only one) allowed 2-body decay mode, namely $\tilde{\ell} \rightarrow \tilde{N}_{1} \ell$. This decay is never strongly suppressed 
because $\tilde{N}_{1}$ always has a significant gaugino component. Therefore, charged slepton production will essentially always give rise to the signal $\ell^{+} \ell^{-} \gamma \gamma \notin$, while sneutrino production, like $\tilde{N}_{1} \tilde{N}_{1}$ production, can give rise only to $\gamma \gamma \not$ E. The leptons appearing in $\gamma \gamma \ell^{+} \ell^{-} \mathbb{E}$ events from charged slepton pair production at LEP should necessarily be quite soft, because there cannot be a large mass difference between the slepton and $\tilde{N}_{1}$ and the sleptons cannot have a large boost. However, the SM backgrounds for such processes are extremely small. Taking into account the cuts (39), (40), one finds that $\gamma \gamma Z Z$ production is always below threshold at LEP160, LEP175, and LEP190, while $\gamma \gamma W W$ is only above threshold at LEP190. Using CompHEP we have found that the latter process only contributes about $0.1 \mathrm{fb}$ to the $\ell \ell \gamma \gamma \mathbb{E}$ background at $\sqrt{s}=190$ $\mathrm{GeV}$. There is also a background for ee $\gamma \gamma \notin$ and $\mu \mu \gamma \gamma \not$ from the process $e^{+} e^{-} \rightarrow Z^{(*)} \gamma \gamma$ with $Z \rightarrow \tau^{+} \tau^{-}$and leptonic $\tau$ decays, but this is very small. Similarly, the photons produced in sneutrino pair production should be softer than those found in $\tilde{N}_{1} \tilde{N}_{1}$ events. Since slepton interactions are not expected to exhibit significant flavor violation, we can conclude by noting that the signatures for the gravitino LSP scenario at LEP are always $\gamma \gamma \notin$ (from $\tilde{N}_{1} \tilde{N}_{1}$ and $\tilde{\nu} \tilde{\nu}$ production) and $e^{+} e^{-} \gamma \gamma \mathbb{E}, \mu^{+} \mu^{-} \gamma \gamma \mathbb{E}$, and $\tau^{+} \tau^{-} \gamma \gamma \mathbb{E}$ (from charged slepton production).

\section{Concluding Remarks}

In this paper, we have studied discovery signals for supersymmetry with a gravitino LSP at the Tevatron and at LEP. If the decay $\tilde{N}_{1} \rightarrow \gamma \tilde{G}$ occurs within the detector, then supersymmetric phenomenology at colliders will have a very bright future. Indeed, the existing Tevatron data of $100 \mathrm{pb}^{-1}$ should allow the exclusions $m_{\tilde{N}_{1}}>70 \mathrm{GeV}$ and $m_{\tilde{C}_{1}}>125 \mathrm{GeV}$ at least in models obeying the gaugino mass unification condition (4). For $m_{\tilde{N}_{1}}>50 \mathrm{GeV}$, it should be possible to exclude $m_{\tilde{C}_{1}}<100 \mathrm{GeV}$ in a model-independent way. The reach is much higher. These results rely on the fact that every supersymmetric event contains two potentially detectable energetic photons and $\mathbb{E}_{T}$, yielding a high (up to $30 \%$ ) detection efficiency. We emphasize that this efficiency is not expected to be significantly reduced by small mass splittings between charginos and neutralinos, since both the photon energies and the $\mathbb{E}_{T}$ depend only on the mass and boost of the $\tilde{N}_{1}$. If the single CDF ee $\gamma \gamma \mathbb{E}_{T}$ event is an example of such an event, then it is not unlikely that an upgraded Tevatron with $\sqrt{s}=2 \mathrm{TeV}$ and $\geq 2 \mathrm{fb}^{-1}$ of data can establish a discovery. In any case, Tevatron upgrades will continue to make strong inroads into the parameter space of the gravitino LSP scenario. The reach and exclusion capability can be estimated for Tevatron upgrades using Figs. 11 and 2 and assuming efficiencies $\gtrsim 15 \%$ as found in Figs. 5 and 6. (Note that the increase in $\sqrt{s}$ from $1.8 \mathrm{TeV}$ to $2 \mathrm{TeV}$ makes such estimates conservative.) 
It is quite possible that LEP190 with $500 \mathrm{pb}^{-1}$ of data can make the discovery if the lightest neutralino is kinematically accessible. At least in the case of models not already excludable by the Tevatron with gaugino mass unification, only $\tilde{N}_{1} \tilde{N}_{1}$ and slepton pair-production can be ex-

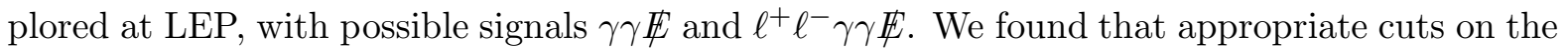
missing invariant mass and on photon energies can reduce the $\gamma \gamma \notin$ backgrounds to a negligible level while keeping intact at least $50 \%$ of the signal even in the worst kinematic situation. The discovery reach extends to within a few $\mathrm{GeV}$ of the kinematic limit. An important factor in the $e^{+} e^{-} \rightarrow \tilde{N}_{1} \tilde{N}_{1} \rightarrow \gamma \gamma$ स search is the mass of the right-handed selectron. If $m_{\tilde{e}_{R}} \lesssim 175 \mathrm{GeV}$, then there should be at least 10 events after cuts in $500 \mathrm{pb}^{-1}$ at $\sqrt{s}=190 \mathrm{GeV}$ for $m_{\tilde{N}_{1}} \lesssim 85$ $\mathrm{GeV}$, but the rate can be much lower for larger $m_{\tilde{e}_{R}}$. In this sense, any exclusion limits will be dependent on assumed upper bounds for $m_{\tilde{e}_{R}}$. If $\tilde{e}_{R}$ is light, then LEP160 and LEP175 can observe a few events.

Although we have not studied future colliders here, it seems clear that both the Large Hadron Collider and Next Linear Collider will be very effective discovery machines if the detectors have good efficiency for detecting isolated energetic photons and $\mathbb{E}_{T}$. If the reported $e e \gamma \gamma \mathbb{E}_{T} \mathrm{CDF}$ event is interpreted as slepton or chargino production, it seems essentially certain that the NLC will detect supersymmetric events, and that the LHC also will if the detectors are sufficiently good with photons and $\mathbb{E}_{T}$.

In general, the ability of the present Tevatron data sample to bound the gravitino LSP scenario emphasizes the importance of photon detection. This component should not be ignored in future detector design. Also, it would be useful to have photon pointing information, in case the $\tilde{N}_{1} \rightarrow \gamma \tilde{G}$ decay length is macroscopic. As we mentioned in section 2 , it is certainly possible that this is so, leading to more single photon events than diphoton events. In that case, one can imagine discovering supersymmetry using the usual well-known discovery signals of the neutralino LSP scenario, supplemented by a fraction of these events with one additional energetic photon. Measuring this fraction would provide a powerful piece of information in disentangling the signal, especially if it can be combined with measurements of the $\tilde{N}_{1}$ decay length.

The gravitino LSP possibility also provides a rich area for theoretical explorations. This scenario necessarily implies a low scale of spontaneous supersymmetry breaking, but the precise mechanism for this breaking and its communication to the fields of the MSSM remain open questions [3]. The connections between supersymmetry breaking and the $\mu$ parameter and other aspects of electroweak symmetry breaking are also interesting questions [32]. We should also mention that if the gravitino is the LSP, then the lightest neutralino is of course no longer a cold dark matter candidate. It remains to be seen if one can obtain a viable dark matter scenario; for a recent proposal see [33]. It seems clear that such issues merit further investigation. 


\section{Acknowledgments}

We are indebted to Bob Blair, Marty Einhorn, Henry Frisch, Tony Gherghetta, Joey Hus-

ton, and Eve Kovacs for helpful discussions. This work was supported in part by the U.S. Department of Energy.

\section{References}

[1] P. Fayet, Phys. Lett. B 70 (1977) 461; Phys. Lett. B 86 (1979) 272; Phys. Lett. B 175 (1986) 471; and in "Unification of the fundamental particle interactions", eds. S. Ferrara, J. Ellis, P. van Nieuwenhuizen (Plenum, New York, 1980) p. 587.

[2] M. Dine, W. Fischler, M.Srednicki, Nucl. Phys. B189 (1981) 575; S. Dimopoulos, S. Raby, Nucl. Phys. B192 (1981) 353; M. Dine, W. Fischler, Phys. Lett. B 110 (1982) 227; M. Dine, M. Srednicki, Nucl. Phys. B202 (1982) 238; L. Alvarez-Gaumé, M. Claudson, M. Wise, Nucl. Phys. B207 (1982) 96; C. Nappi, B. Ovrut, Phys. Lett. B 113 (1982) 175; M. Dine, W. Fischler, Nucl. Phys. B204 (1982) 346; S. Dimopoulos, S. Raby, Nucl. Phys. B219 (1983) 479.

[3] For recent examples see M. Dine, A. E. Nelson, Phys. Rev. D48 (1993) 1277; M. Dine, A. E. Nelson, Y. Shirman, Phys. Rev. D51 (1995) 1362; M. Dine, A. E. Nelson, Y. Nir, Y. Shirman, Phys. Rev. D53 (1996) 2658.

[4] A. E. Nelson, N. Seiberg, Nucl. Phys. B416 (1994) 46; J. Bagger, E. Poppitz, L. Randall, Nucl. Phys. B426 (1994) 3.

[5] T. Bhattacharya, P. Roy, Phys. Lett. B 206 (1988) 655; Nucl. Phys. B328 (1989) 481.

[6] H. Pagels, J. R. Primack, Phys. Rev. Lett. 48 (1982) 223; T. Moroi, H. Murayama, M. Yamaguchi, Phys. Lett. B 303 (1993) 289.

[7] For a review, see H. Baer et al., "Low-energy supersymmetry phenomenology", hepph/9503479.

[8] N. Cabibbo, G. R. Farrar, and L. Maiani, Phys. Lett. B 105 (1981) 155; M. K. Gaillard, L. Hall, I. Hinchliffe, Phys. Lett. B 116 (1982) 279; J. Ellis, J. S. Hagelin, Phys. Lett. B 122 (1983) 303; D. A. Dicus, S. Nandi, and J. Woodside, Phys. Lett. B 258 (1991) 231.

[9] D. R. Stump, M. Wiest, C. P. Yuan, preprint hep-ph/9601362.

[10] K. Kiers, J. N. Ng, G. Wu, preprint hep-ph/9604338.

[11] S. Dimopoulos, S. Thomas, J. D. Wells, preprint hep-ph/9604452.

[12] S. Park, "Search for New Phenomena in CDF", 10 ${ }^{\text {th }}$ Topical Workshop on Proton-Antiproton Collider Physics, edited by Rajendran Raja and John Yoh, AIP Press, 1995. 
[13] S. Dimopoulos, M. Dine, S. Raby, S. Thomas, Phys. Rev. Lett. 76 (1996) 3494.

[14] S. Ambrosanio, G. L. Kane, G. D. Kribs, S. P. Martin, S. Mrenna, Phys. Rev. Lett. 76 (1996) 3498.

[15] E. Cremmer, S. Ferrara, L. Girardello, A. van Proyen, Nucl. Phys. B212 (1983) 413.

[16] H. E. Haber, G. L. Kane, Phys. Rep. 117 (85) 75; J. F. Gunion, H. E. Haber, Nucl. Phys. B272 (1986) 1; Erratum ibid B402 (1993) 567.

[17] J. M. Cornwall, D. N. Levin, G. Tiktopoulos, Phys. Rev. D10 (1974) 1145, Erratum ibid D11 (1975) 972.

[18] D. A. Dicus, S. Nandi, J. Woodside, Phys. Rev. D41 (1990) 2347.

[19] M. Drees, J. Woodside in Supersymmetry, Proc. of ECFA Workshop on the Large Hadron Collider, 1990.

[20] M. Drees, J. Ellis, P. Jetzer, D. W. Sciama, Phys. Lett. B 220 (1989) 586

[21] H.U. Bengtsson, T. Sjöstrand, Comp. Phys. Comm. 46 (1987) 43; T. Sjöstrand, Comp. Phys. Comm. 82 (1994) 74; S. Mrenna, "Simulating Supersymmetry With PYTHIA 5.7 and JETSET 7.4," CITHE-68-1987, July 1995.

[22] See for example H. Baer, C.-h. Chen, C. Kao, X. Tata, Phys. Rev. D52 (1995) 1565; S. Mrenna, G.L. Kane, G.D. Kribs, J.D. Wells, Phys. Rev. D53 (1996) 1168.

[23] We thank Bob Blair (Argonne) for providing information on the CDF diphoton crosssection measurements.

[24] We thank T. Sjöstrand (Lund) for this suggestion.

[25] H. Baer, C.-h. Chen, F. Paige, X. Tata, Phys. Rev. D49 (1994) 3283.

[26] A. Djouadi et al. Nucl. Phys. B349 (1991) 48; M. Boulware, D. Finnell, Phys. Rev. D44 (1991) 2054; J. D. Wells, C. Kolda, G. L. Kane, Phys. Lett. B 338 (1994) 219; D. Garcia, J. Sola, Phys. Lett. B 354 (1995) 335; G. L. Kane, R. G. Stuart, J. D. Wells, Phys. Lett. B 354 (1995) 350; A. Dabelstein, W. Hollik, W. Mösle, hep-ph/9506251; P. H. Chankowski, S. Pokorski, Phys. Lett. B 366 (1996) 188; J. Ellis, J. Lopez, D. Nanopoulos, Phys. Lett. B 372 (1996) 95; J. D. Wells, G. L. Kane, Phys. Rev. Lett. 76 (1996) 869; E. H. Simmons, Y. Su, hep-ph/9602267; P. H. Chankowski, S. Pokorski, hep-ph/9603310.

[27] D.R. Claes, $10^{\text {th }}$ Topical Workshop on Proton-Anti-proton Collider Physics, edited by Rajendran Raja and John Yoh, AIP Press, 1995, FERMILAB-CONF-95/186-E.

[28] S. P. Martin, M. T. Vaughn, Phys. Lett. B 318 (1993) 331. The corresponding (smaller) corrections for charginos and neutralinos are given in D. Pierce and A. Papadopoulos, Phys. Rev. D50 (1994) 565, Nucl. Phys. B430 (1994) 278.

[29] H. Baer, C.-h. Chen, C. Kao, and X. Tata, Phys. Rev. D52 (1995) 1565. 
[30] F. Boudjema, B. Mele (Conveners), E. Accomando et al. [The Standard Model Process Group], Proc. of the Workshop "Physics at LEP2", G. Altarelli, T. Sjöstrand, F. Zwirner (eds.), CERN-Report 96-01 (1996), hep-ph/9601224.

[31] E. E. Boos, M. N. Dubinin, V. A. Ilin, A. E. Pukhov, V. I. Savrin, "CompHEP: Specialized package for automatic calculation of elementary particle decays and collisions", hep-ph/9503280, and references therein.

[32] G. Dvali, G. F. Giudice, A. Pomarol, hep-ph/9603238.

[33] S. Borgani, A. Masiero, M. Yamaguchi, hep-ph/9605222. It is not clear that the requirements of this paper can be satisfied if the CDF ee $\gamma \gamma \mathbb{E}_{T}$ event is interpreted as superpartner production. 